\title{
Adipocyte-specific deletion of Ip6k1 reduces diet-induced obesity by enhancing AMPK-mediated thermogenesis
}

\author{
Qingzhang Zhu, ${ }^{1}$ Sarbani Ghoshal, ${ }^{1}$ Ana Rodrigues, ${ }^{1}$ Su Gao, ${ }^{1}$ Alice Asterian, ${ }^{2}$ Theodore M. Kamenecka, ${ }^{2}$ \\ James C. Barrow, ${ }^{3}$ and Anutosh Chakraborty ${ }^{1}$ \\ 'Department of Metabolism and Aging, and ${ }^{2}$ Department of Molecular Therapeutics, The Scripps Research Institute, Jupiter, Florida, USA. ${ }^{3}$ Drug Discovery Division, Lieber Institute \\ for Brain Development, Baltimore, Maryland, USA
}

\begin{abstract}
Enhancing energy expenditure (EE) is an attractive strategy to combat obesity and diabetes. Global deletion of Ip6k1 protects mice from diet-induced obesity (DIO) and insulin resistance, but the tissue-specific mechanism by which IP6K1 regulates body weight is unknown. Here, we have demonstrated that IP6K1 regulates fat accumulation by modulating AMPK-mediated adipocyte energy metabolism. Cold exposure led to downregulation of Ip6k1 in murine inguinal and retroperitoneal white adipose tissue (IWAT and RWAT) depots. Adipocyte-specific deletion of Ip6k1 (AdKO) enhanced thermogenic EE, which protected mice from high-fat diet-induced weight gain at ambient temperature $\left(23^{\circ} \mathrm{C}\right)$, but not at thermoneutral temperature $\left(30^{\circ} \mathrm{C}\right)$. AdKO-induced increases in thermogenesis also protected mice from cold-induced decreases in body temperature. UCP1, PCC1 $\alpha$, and other markers of browning and thermogenesis were elevated in IWAT and RWAT of AdKO mice. Coldinduced activation of sympathetic signaling was unaltered, whereas AMPK was enhanced, in AdKO IWAT. Moreover, beige adipocytes from AdKO IWAT displayed enhanced browning, which was diminished by AMPK depletion. Furthermore, we determined that IP6 and IP6K1 differentially regulate upstream kinase-mediated AMPK stimulatory phosphorylation in vitro. Finally, treating mildly obese mice with the IP6K inhibitor TNP enhanced thermogenesis and inhibited progression of DIO. Thus, IP6K1 regulates energy metabolism via a mechanism that could potentially be targeted in obesity.
\end{abstract}

\section{Introduction}

Obesity and type 2 diabetes (T2D) lead to several comorbid conditions, such as coronary heart disease, stroke, fatty liver, neurodegeneration, and various other diseases. A shift of the equilibrium toward energy expenditure (EE) over energy intake ameliorates obesity (1). Stored energy is utilized by respiration that generates ATP or by uncoupling protein 1-mediated (UCP1-mediated) thermogenesis, which releases heat. Brown adipose tissue (BAT) expends energy primarily by thermogenesis (2). Moreover, white adipocytes can transform into brown-like thermogenic "beige" or "brite" cells under certain stimulatory conditions $(3,4)$. Thus, an enhancement in white adipose tissue (WAT) browning augments EE, which facilitates fat loss in rodents and humans (4-7). Functional BAT in adult humans $(2,8)$ efficiently takes up glucose (18F-fluoro-2-deoxy-D-glucose) (8, 9). Moreover, metabolically active adult BAT exhibits an inverse correlation with BMI, adiposity, and insulin resistance, and thus, targeting BAT is an attractive therapeutic strategy in obesity (10-14). Likewise, beige adipocytes have also attracted much attention as an antiobesity target, due to their inducible nature and relevance to adult humans $(1,2$, $4,5,8,15)$. Cold exposure, sympathetic transmission, thyroid hormone, and cardiac natriuretic peptides induce UCP1-mediated thermogenesis via stimulation of the $\beta$-adrenergic receptormediated ( $\beta$-AR-mediated) cAMP/PKA pathway, which primarily activates the transcriptional coactivator PPAR $\gamma$ coactivator $1-\alpha$

Conflict of interest: The authors have declared that no conflict of interest exists. Submitted: November 11, 2015; Accepted: August 29, 2016.

Reference information: / Clin Invest. 2016;126(11):4273-4288. doi:10.1172/JCI85510.
(PGC1- $\alpha)(5,16)$ PGC1- $\alpha$ promotes respiration, thermogenesis, and mitochondrial biogenesis by stimulating several transcription factors and coactivators $(4,17,18)$.

Cold and energy stress also induce the stress-activated protein kinase AMP-activated protein kinase (AMPK), which enhances $\mathrm{EE}$ and thus is a major target in obesity and T2D (19-24). $\beta$-ARmediated AMPK activation augments insulin sensitivity in a UCP1dependent manner (22). Conversely, prolonged inhibition of adrenergic receptors decreases AMPK activity (25). Accordingly, the plant-derived alkaloid berberine or microRNA-455 enhances brown adipogenesis via activation of $\operatorname{AMPK}(26,27)$. In addition, AMPK stimulates glycolysis (28) and coupled respiration-mediated ATP generation in metabolic tissues (29-31). Hence, the AMPK activator 5-aminoimidazole-4-carboxamide $1-\beta$-D-ribofuranoside (AICAR) stimulates EE $(32,33)$. AMPK enhances EE by modulating various targets, of which PGC1- $\alpha$ and acetyl-CoA carboxylase (ACC) are the most characterized $(21,34)$. AMPK consists of the catalytic $\alpha$ and the regulatory $\beta / \gamma$ subunits (35). During stress, LKB1 or CaMKK $\beta$ phosphorylates AMPK $\alpha$ at the threonine 172 residue, which stimulates its catalytic activity $(21,36,37)$. Yet a complete knowledge of AMPK regulation, especially in metabolic tissues, under various temperature and diet conditions, is lacking (37).

Inositol phosphates have been appreciated as biologically relevant molecules since the identification of inositol hexakisphosphate (IP6 or phytic acid) as an abundant phosphate-storage constituent of plants. The most appreciated inositol phosphate is the second messenger IP3 [Ins(1,4,5) P3], which releases calcium from intracellular stores (38). Inositol polyphosphate derivatives with highly energetic diphosphates (inositol pyrophosphates; IP7) 
were identified in the mid-1990s (39, 40). 5-IP7 (5-diphosphoinositol pentakisphosphate) is the major IP7 isoform in mammals (41), which is synthesized by a family of 3 IP6 kinases (IP6Ks) (42). At lower ATP/ADP ratio, IP6Ks dephosphorylate IP6 to a distinct form of IP5 [Ins $(2,3,4,5,6)$ P5, IP5 ${ }^{*}$ (43). IP6 regulates cellular processes by binding to its protein targets (44), whereas 5-IP7 modulates its targets by binding or by pyrophosphorylation $(42,45-48)$. IP6Ks also regulate cellular functions via distinct mechanisms that do not require catalytic activity (49-52). For instance, IP6K1 interacts with glycogen synthase kinase and perilipin to modulate their cellular functions $(49,52)$.

Functions of 3 mammalian IP6K isoforms (IP6K1-3) are being explored. Although IP6Ks display conserved active sites, they possess unique regulatory regions, which mediate specific proteinprotein interactions $(45,53)$. Accordingly, IP6Ks exhibit distinct cellular functions $(45,54)$. IP6K1-generated 5-IP7 modulates cell metabolism, signaling, exocytosis, and migration (48, 55-58). IP6K1-generated 5-IP7 regulates insulin signaling by inhibiting the insulin-sensitizing protein kinase Akt both in vivo and in vitro (58-61), whereas it promotes insulin secretion from pancreatic $\beta$ cells (57). IP6K1 also regulates global transcription by 5-IP7mediated inhibition of the histone demethylase JMJD2C (62). In immortalized mouse embryonic fibroblast (MEF) cells, IP6K1generated 5-IP7 reduces cellular ATP level and lowers the glycolytic/oxidative phosphorylation ratio (55).

The compound TNP [N2-(m-trifluorobenzyl),N6-( $p$-nitrobenzyl) purine] (63) inhibits IP6Ks (64), whereas it does not influence the other IP7-generating (1-IP7-generating) enzyme PP-IP5K (65) or 71 unrelated kinases (64). Thus, TNP is a specific IP6K inhibitor. Accordingly, TNP reduces intracellular 5-IP7 levels and activates Akt $(45,58,59,61,64,66)$. TNP-mediated inhibition of IP6Ks also leads to a corresponding increase in the intracellular concentration of the IP6K substrate IP6 $(58,67)$. Recent studies indicate that TNP-mediated IP6K inhibition has functional significance in vivo. For instance, 5-IP7 level is increased in aged mesenchymal stem cells (61). TNP reduces 5-IP7, which stimulates Akt-mediated survival of these cells (61). Moreover, TNP treatment leads to a substantial reduction in 5-IP7 levels in the hearts of diabetic $d b / d b$ mice (66). Accordingly, TNP protects cardiac ischemia in these mice via enhancement of Akt-mediated survival and AMPK/ PGC1- $\alpha$-mediated mitochondrial biogenesis (66).

In order to understand the physiological role of IP6K1, mice with this gene globally deleted were generated (68). Deletion of Ip6k1 significantly influences various aspects of mouse biology, and thus, targeting this isoform is expected to be beneficial in obesity, T2D $(45,58,69,70)$, innate immunity (59), thromboembolism (71), cancer metastasis (56), and psychiatric and neurodegenerative diseases (49). Ip6k1 KO mice are protected against high-fat diet-induced (HFD-induced) weight gain, insulin resistance, and fatty liver (58). HFD-fed Ip6k1 KOs maintain insulin sensitivity by sustaining Akt activity (58). Moreover, HFD-fed Ip6k1 KO mice expend more energy, whereas their energy intake is unaltered (58). Yet the precise tissue-specific mechanism by which IP6K1 regulates energy homeostasis is not known. To determine this, we generated an adipocyte-specific Ip6k1 KO mouse model (AdKO). Using this model, we demonstrate that IP6K1 regulates global energy homeostasis by modulating AMPK-mediated adipose tis- sue browning and thermogenesis. In addition to its inhibitory actions on Akt, IP6K1 reduces AMPK activity in the adipose tissue, although via a distinct and novel mechanism.

\section{Results}

Adipocyte-specific Ip6k1-deleted mice accumulate less fat because of enhanced thermogenic EE under chow-fed conditions. Adipocytespecific Ip $6 k 1 \mathrm{KO}$ (AdKO) mice are generated by crossing of Ip6k1LoxP mice (68) with Adipoq-Cre mice following standard procedure (Figure 1A). IP6K1 protein level is dramatically reduced in diverse adipose tissue depots of AdKOs (Figure 1B). Ip6k2 or Ip6k3 does not compensate for Ip6k1 deletion in the AdKO adipose tissue (Supplemental Figure 1A; supplemental material available online with this article; doi:10.1172/JCI85510DS1). Chow diet-fed AdKOs (CD-AdKOs), at a young age (3-4 weeks), do not display any variation in body weight, which indicates that the knockouts do not exhibit developmental defects in weight gain (Supplemental Figure 1B). At 2 months, AdKOs display a marginal decrease in fat mass (Figure $1 \mathrm{C}$ and Supplemental Figure 1C), whereas lean and fluid masses are unchanged (Supplemental Figure 1, D and E). Accordingly, weights of various adipose depots and their adipocyte sizes are marginally less in these mice (Figure 1D and Supplemental Figure 1F). Weights of other tissues such as liver and heart are similar (Supplemental Figure 1G). Food intake is comparable in CD-fed LoxPs and AdKOs (data not shown). Conversely, CD-AdKOs exhibit enhanced $\mathrm{VO}_{2} / \mathrm{EE}$ at $23^{\circ} \mathrm{C}$ (Figure $1, \mathrm{E}$ and $\mathrm{F}$ ). We also measured these parameters at $5^{\circ} \mathrm{C}$ ( 24 hours) and acute fasting (6 hours) at $5^{\circ} \mathrm{C}$ (to enhance expenditure of stored energy to maintain body temperature). CD-AdKOs exhibit higher $\mathrm{VO}_{2} / \mathrm{EE}$ under these conditions (Figure 1, E and G). Respiratory exchange ratio (RER) is slightly higher in CD-AdKOs at $23^{\circ} \mathrm{C}$ but not at $5^{\circ} \mathrm{C}$ (Supplemental Figure $1 \mathrm{H}$ ). Further analyses (72) reveal that $\mathrm{CD}$-AdKOs consume more oxygen for carbohydrate oxidation at $23^{\circ} \mathrm{C}$ and $5^{\circ} \mathrm{C}$ whereas they oxidize more fat when exposed to cold plus fasting conditions (Figure $1, \mathrm{H}$ and I). Activity profiles are unaltered (Supplemental Figure 1I). Enhanced thermogenic EE protects CD-AdKOs from both acute- and chronic-cold-induced decline in body temperature (Figure 1, J and K). Further analyses indicate that $I p 6 k 1$ is the major isoform in various adipose depots of WT C57BL/6 mice (Figure 1L). Moreover, Ip6k1 expression is reduced in inguinal WAT (IWAT) and retroperitoneal WAT (RWAT) but not in BAT following acute cold exposure (Figure 1L). Similarly to acute, chronic cold exposure also reduces Ip6k1 expression in the IWAT, whereas Ip6k2 and Ip6k3 levels are unaltered (Figure $1 \mathrm{M}$ ). These results suggest that Ip6k1, the major Ip $6 k$ isoform in the adipose tissue, inhibits thermogenic EE.

Increased thermogenic EE decelerates HFD-induced weight gain in AdKO mice. At $23^{\circ} \mathrm{C}$, AdKOs are protected against HFD-induced weight gain, which is apparent from 2 weeks and becomes significant after 4 weeks (Figure 2A and Supplemental Figure 2A). After 14 weeks of HFD, AdKOs appear substantially smaller, because of less fat accumulation (Figure 2B). In HFD-AdKOs, total fat mass is reduced $(\Delta 4.52 \mathrm{~g})$ to a greater extent than lean mass ( $\Delta$ $2.46 \mathrm{~g}$ ) (Figure 2C). Percentage fat mass (of total body weight) is also reduced in HFD-AdKOs, whereas percentage lean mass is increased (Supplemental Figure 2B). Accordingly, weights of diverse adipose depots are significantly less in HFD-AdKOs (Fig- 

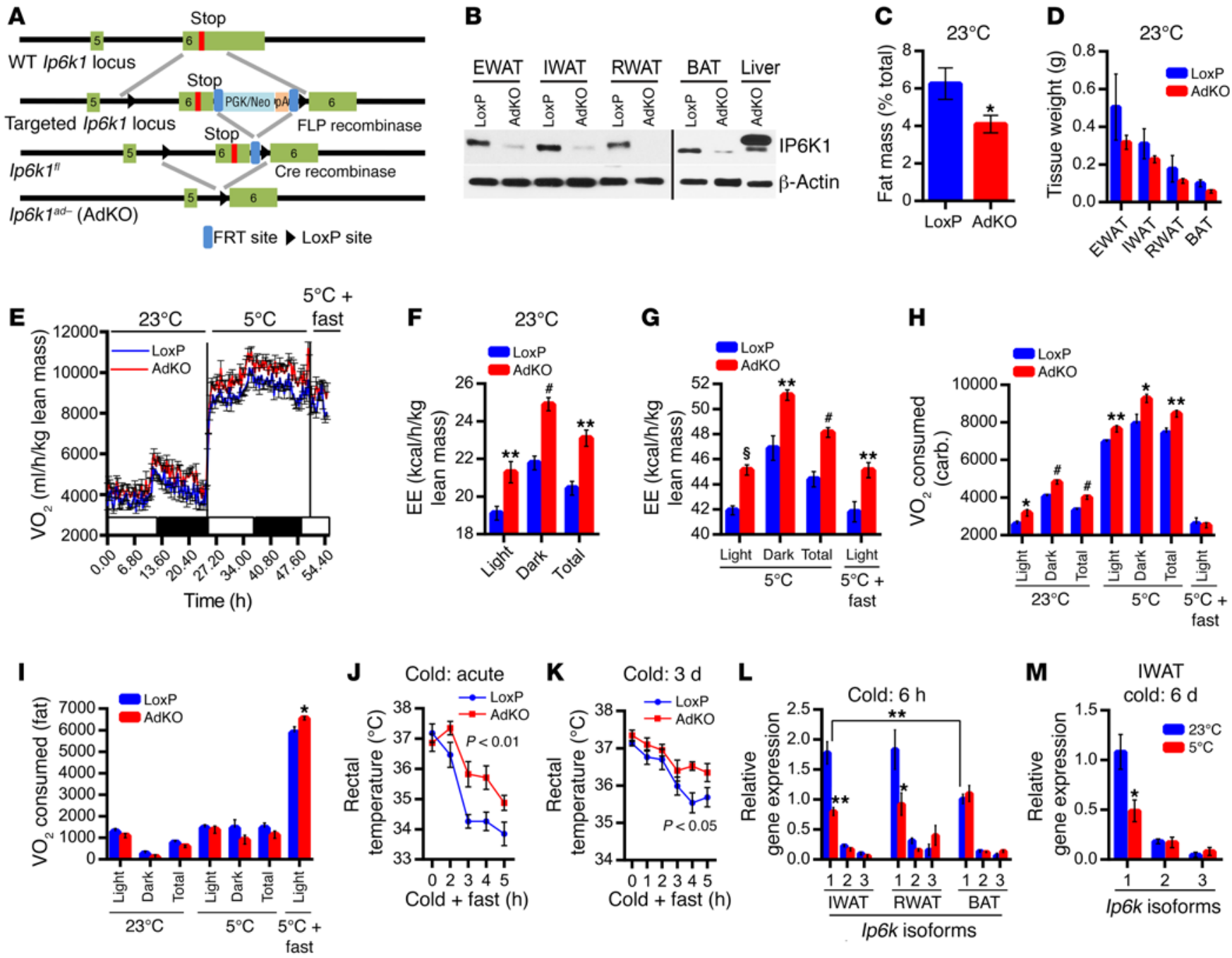

Figure 1. Adipocyte-specific Ip6k1-deleted mice accumulate less fat because of enhanced thermogenic EE under chow-fed conditions. (A) Ceneration of AdKOs. Details of the knockout generation strategy are described in Methods. (B) Immunoblot analyses confirm deletion of IP6K1 protein from various adipose tissue depots. Detection of residual IP6K1 is due to the presence of macrophages and other immune cells in the adipose tissue. Liver samples from AdKO mice were run as a control. BAT and liver samples were run on a separate gel. Blot represents data from 3 independent experiments. (C) CDAdKOs ( 8 weeks old) display significantly reduced percentage fat mass ( $n=10$ mice per group; $t$ test). (D) Weights of diverse adipose tissue depots are marginally less in CD-AdKOs ( $n=4$ mice per group; $t$ test). (E) VO $\mathrm{V}_{2}$ is higher in CD-AdKOs at $23^{\circ} \mathrm{C}$ and following cold and cold + fasting exposures ( $n=8$ mice per group). ( $F$ and $\mathbf{G}) \mathrm{EE}$ is higher in $\mathrm{CD}$-AdKOs under the above-mentioned conditions ( $n=8$ mice per group; $t$ test). (H) CD-AdKOs consume more oxygen to oxidize carbohydrate at $23^{\circ} \mathrm{C}$ and $5^{\circ} \mathrm{C}\left(n=8\right.$ mice per group; $t$ test). (I) Following acute fasting at $5^{\circ} \mathrm{C}$, CD-AdKOs consume more oxygen for fat oxidation ( $n=8$ mice per group; $t$ test). (J) CD-AdKOs maintain body temperature more efficiently than LoxPs following acute cold + fasting exposure ( $n=8$ mice per group; 2-way ANOVA). (K) CD-AdKOs are also partly protected against drop in body temperature induced by chronic cold ( 3 days) and acute cold + fasting ( $n=8$ mice per group; 2-way ANOVA). (L) Ip6k1 is the major Ip6k isoform in the adipose tissue, which is downregulated by acute cold exposure in IWAT and RWAT. Ip6k1 expression is lower in the BAT and is not further reduced by cold ( $n=5$ mice per group; 2 -way ANOVA). (M) Chronic cold also specifically reduces Ip6k1 expression in IWAT ( $n=6$ mice per group; $t$ test). Data in all panels are expressed as mean \pm SEM. ${ }^{*} P<0.05,{ }^{* *} P<0.01,{ }^{\#} P<0.001,{ }^{\S} P<0.0001$.

ure 2D and Supplemental Figure 2C). HFD-AdKOs are protected against adipocyte hypertrophy (Figure 2E, EWAT, IWAT, and RWAT). Moreover, BAT of HFD-AdKOs accumulates substantially less fat (Figure 2E; BAT). In addition, HFD-AdKOs exhibit smaller and healthier liver and are protected against HFD-induced fatty liver (Figure 2F and Supplemental Figure 2D). Weights of other tissues are largely unaltered (Supplemental Figure 2E).

Next, we monitored energy metabolism in HFD-AdKOs. Average daily food intake is slightly (not significantly) higher in HFDAdKOs ( $14.98 \mathrm{kcal})$ compared with LoxPs $(\sim 12.23 \mathrm{kcal})$ (Supplemental Figure $2 \mathrm{~F}$ ). Daily meal size and meal numbers are largely similar in HFD-fed LoxPs and AdKOs (Supplemental Figure 2, G and $\mathrm{H}$ ). Conversely, $\mathrm{VO}_{2}$ and $\mathrm{EE}$ are significantly higher in AdKOs at $23^{\circ} \mathrm{C}$ (Figure $2 \mathrm{G}$ and Supplemental Figure 2, I and J, $23^{\circ} \mathrm{C}$ ). Interestingly, $\mathrm{VO}_{2}$ and $\mathrm{EE}$ are further induced in cold-exposed HFDAdKOs (Figure 2, G and H, and Supplemental Figure 2, I and K, $5^{\circ} \mathrm{C}$ and $5^{\circ} \mathrm{C}+$ fast). RER is higher in HFD-AdKOs at $23^{\circ} \mathrm{C}$ but not at $5^{\circ} \mathrm{C}$ (Supplemental Figure 2L). Accordingly, HFD-AdKOs consume more oxygen for carbohydrate oxidation at $23^{\circ} \mathrm{C}$ (Figure 2I). Conversely, the knockouts oxidize more fat at $5^{\circ} \mathrm{C}$ (Figure 2J). Activity parameters are unaltered in HFD-AdKOs (Supplemental Figure 2M). Thus, increased thermogenic EE protects AdKOs from 
A

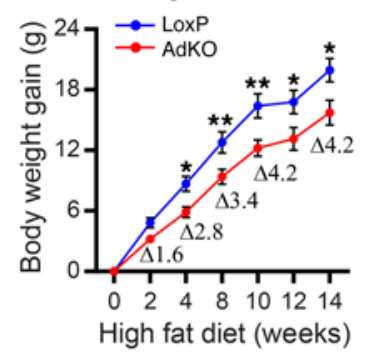

B

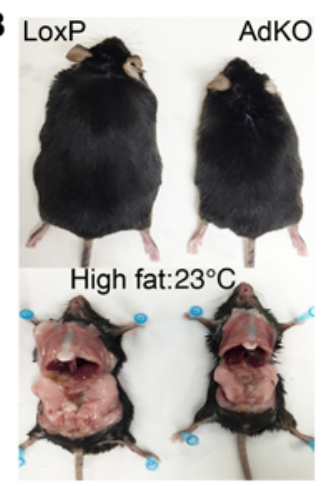

C

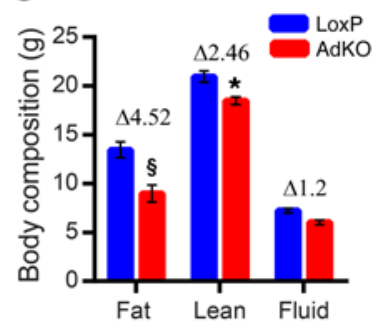

D

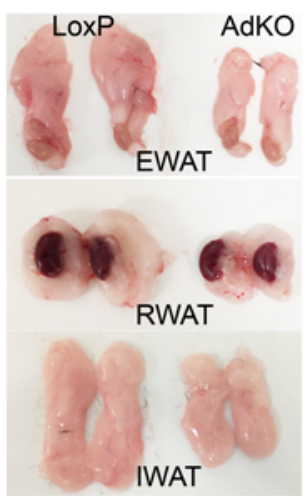

High fat: $23^{\circ} \mathrm{C}$

\section{E}

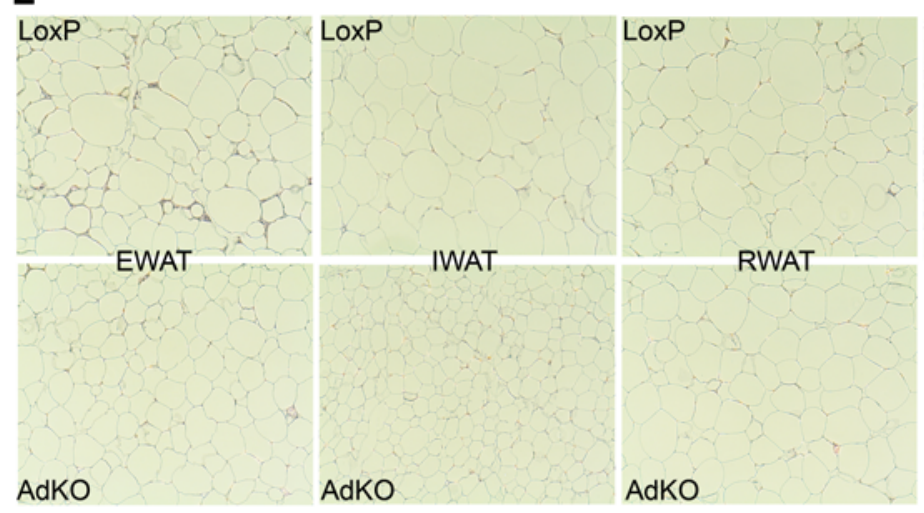

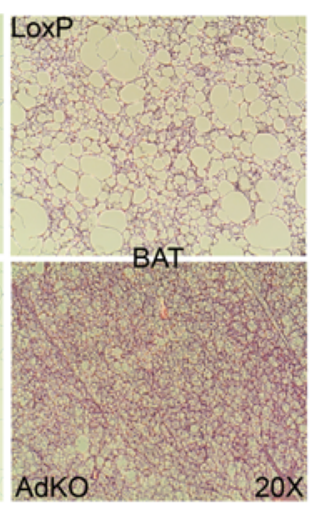

$\mathbf{F}$
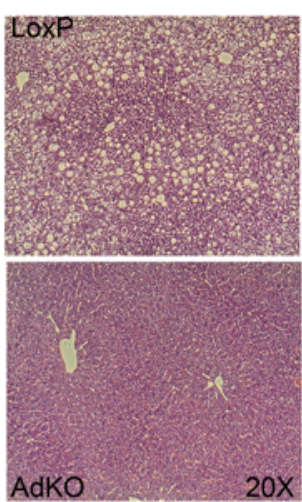

J

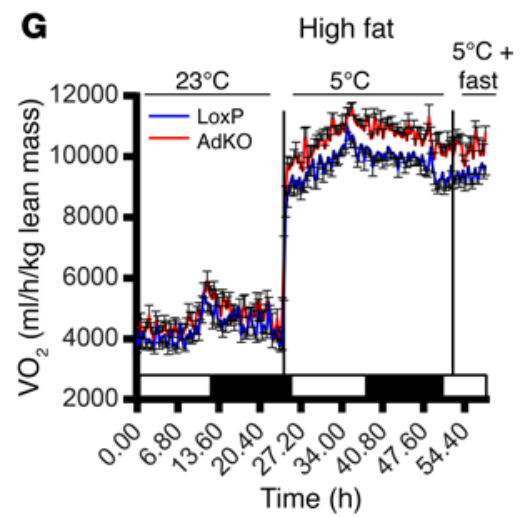

H

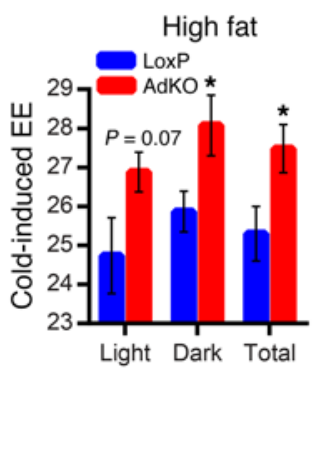

I

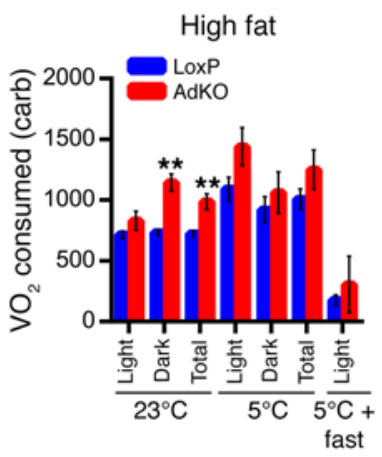

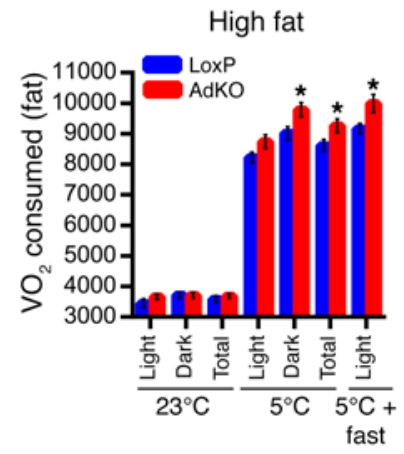

Figure 2. Increased thermogenic EE decelerates HFD-induced weight gain in AdKO mice. (A) Average weekly weight gain is less in HFD-AdKOs ( $n=15$ mice per group; 2-way ANOVA). (B) Representative photographs of mice (from A) demonstrate that after 14 weeks of HFD feeding, AdKOs appear smaller and accumulate less fat. (C) After 8 weeks of HFD feeding, AdKOs display less fat mass. Lean/fluid masses are less altered ( $n=10$ mice per group; $t$ test). (D) Representative photographs (from mice used in A) reveal that EWAT, RWAT, and IWAT appear smaller in HFD-AdKOs. (E) AdKOs are protected against HFD-induced adipocyte hypertrophy. AdKO BAT accumulates less fat (image represents data from $n=3$ mice per group). (F) AdKOs are protected against HFD-induced fatty liver (image represents data from $n=3$ mice per group). (C) AdKOs (8 weeks of $\mathrm{HFD}$ at $23^{\circ} \mathrm{C}$ ) display higher $\mathrm{VO}_{2}$ consumption at $23^{\circ} \mathrm{C}$, which is further enhanced following cold exposure ( $n=8$ mice per group). (H) Average cold-induced EE is higher in HFD-AdKOs ( $n=8$ mice per group; $t$ test). (I) HFD-AdKOs consume more oxygen to oxidize carbohydrate at $23^{\circ} \mathrm{C}$. However, at $5^{\circ} \mathrm{C}$, they oxidize carbohydrate to a similar extent $(n=8$ mice per group; $t$ test). (J) HFD-AdKOs consume similar oxygen for fat oxidation at $23^{\circ} \mathrm{C}$. However, AdKOs oxidize more fat at $5^{\circ} \mathrm{C}$ and following acute fasting at $5^{\circ} \mathrm{C}$ ( $n=8$ mice per group; $t$ test). Data in all panels are expressed as mean \pm SEM. ${ }^{*} P<0.05,{ }^{* *} P<0.01,{ }^{\S} P<0.0001$.

HFD-induced weight gain. Moreover, AdKOs maintain metabolic homeostasis by expending various forms of energy, depending on diet and temperature conditions.

Adipocyte-specific Ip6k1 deletion protects mice from HFDinduced insulin resistance. Blood glucose levels are slightly lower in $\mathrm{CD}$-AdKOs at $23^{\circ} \mathrm{C}$ (Supplemental Figure 3A). However, AdKOs $\left(23^{\circ} \mathrm{C}\right)$ are significantly protected against HFD-induced insulin resistance. Blood glucose level in AdKOs fed HFD for 4 weeks is slightly albeit significantly less in comparison with LoxPs (data not shown), and this is clearly evident after 14 weeks of HFD feeding (Figure 3A). Improved glucose and insulin tolerance tests (GTT and ITT), in mice fed HFD for 14 weeks, demonstrate that HFD- 
A

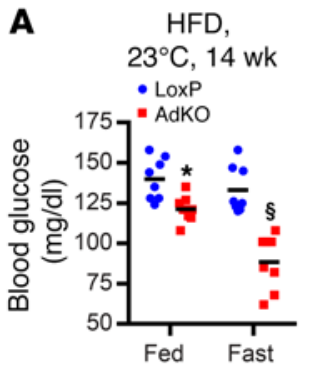

B

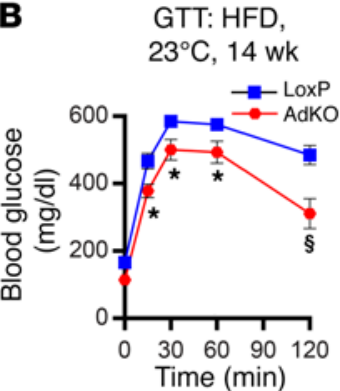

C

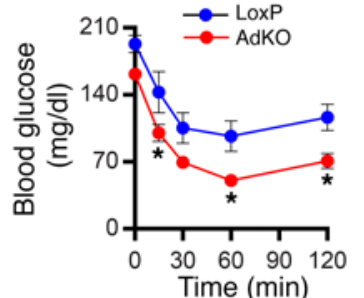

ITT: HFD, $23^{\circ} \mathrm{C}, 14$ wk
D

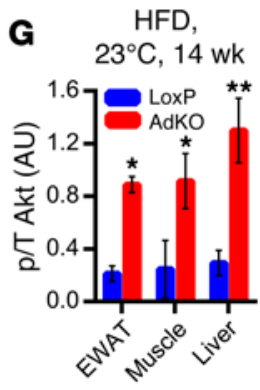

HFD, $23^{\circ} \mathrm{C}, 14$ wk

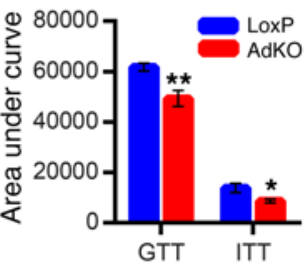

E

HFD, $23^{\circ} \mathrm{C}, 14$ wk

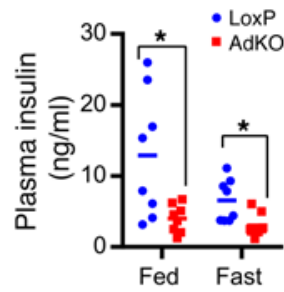

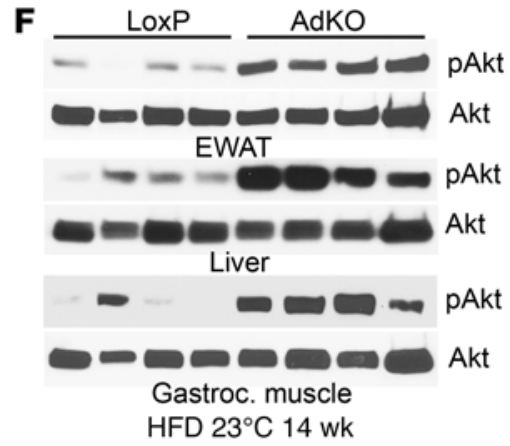

F HFD $23^{\circ} \mathrm{C} 14 \mathrm{wk}$

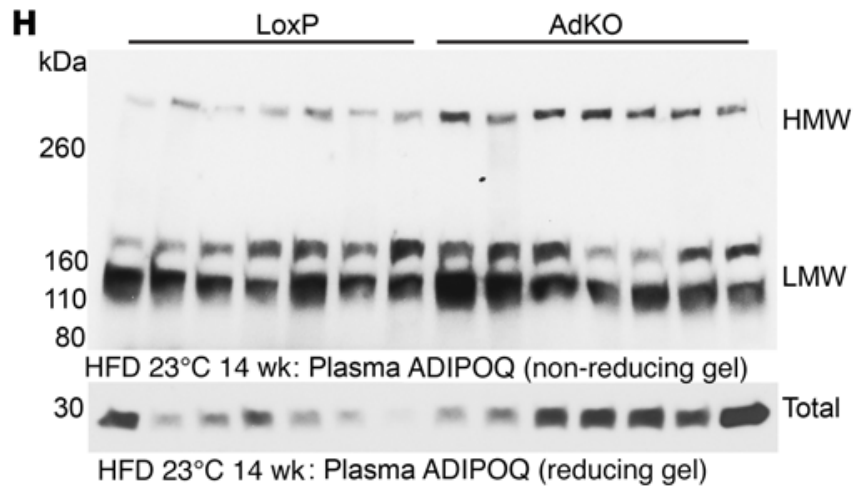

I Plasma: HMW ADIPOQ

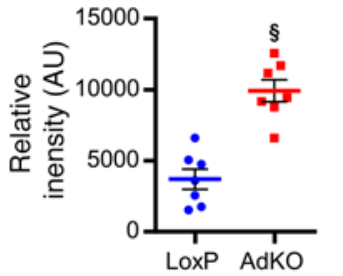

J Plasma: total ADIPOQ

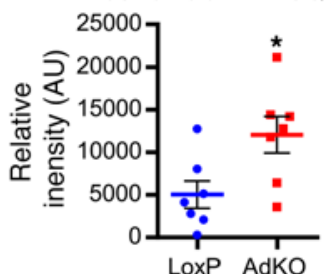

Figure 3. Adipocyte-specific Ip6k1 deletion protects mice from HFD-induced insulin resistance. (A) Blood glucose level is lower in ad libitum HFD-AdKOs. Overnight fasting decreases blood glucose level in HFD-AdKOs but not in corresponding HFD-LoxPs ( $n=8$ mice per group; $t$ test). (B) Improved glucose disposal (GTT) in HFD-AdKOs ( $n=8-10$ mice per group; 2-way ANOVA). (C) Improved glucose disposal in HFD-AdKOs following insulin injection (ITT) ( $n=8-10$ mice per group; 2-way ANOVA). (D) AUC analyses of $\mathbf{B}$ and $\mathbf{C}$ confirm enhanced glucose and insulin tolerances in HFD-AdKOs ( $n=8-10$ mice per group; $t$ test). (E) HFD-AdKOs exhibit reduced plasma insulin level ( $n=8$ mice per group; $t$ test). (F) Akt (S473) stimulatory phosphorylation is substantially higher in the EWAT, liver, and gastrocnemius muscle of HFD-AdKOs ( $n=4$ mice per group). (G) Image) quantification of $\mathbf{F}$ reveals that Akt stimulatory phosphorylation (S473) is 4- to 6-fold higher in EWAT, gastrocnemius muscle, and liver of HFD-AdKOs ( $t$ test). p/T, phosphorylated Akt/total Akt. (H) Plasma levels of HMW and total but not LMW ADIPOQ are higher in HFD-AdKOs ( $n=7$ mice per group). (I) Image) quantification of $\mathbf{H}$ reveals that the plasma level of HMW-ADIPOQ is 2.5-fold higher in HFD-AdKOs ( $t$ test). (J) Imagej quantification of $\mathbf{H}$ demonstrates a 2-fold increase in the plasma level of total ADIPOQ in HFD-AdKOs ( $t$ test). Data in all panels are expressed as mean $\pm \mathrm{SEM}$. ${ }^{*} P<0.05,{ }^{* *} P<0.01,{ }^{\S} P<0.0001$.

AdKOs exhibit improved glucose disposal/insulin hypersensitivity (Figure 3, B-D). Accordingly, HFD-AdKOs display enhanced stimulatory phosphorylation (S473) of Akt in the epididymal white adipose tissue (EWAT), liver, and gastrocnemius muscle, despite reduced plasma insulin level (Figure 3, E-G). Further analyses reveal that CD-fed LoxPs and AdKOs exhibit similar levels of Akt phosphorylation (Supplemental Figure 3B; CD). HFD feeding promotes insulin resistance which reduces Akt phosphorylation in the LoxPs, whereas AdKOs are largely protected (Supplemental Figure 3B; HFD). It is conceivable that adipocyte-specific Ip $6 k 1$ deletion alters expression/secretion of certain adipokines, which enhance insulin sensitivity in other tissues. For example, the insulin sensitizer adiponectin (ADIPOQ), especially its active highmolecular weight (HMW) form, is reduced in obesity and T2D (73). Accordingly, plasma levels of total and HMW ADIPOQ are higher in HFD-AdKOs (Figure 3, H-J, and Supplemental Figure $3 \mathrm{C})$. Thus, adipocyte-specific deletion of $I p 6 k 1$ protects mice from HFD-induced global insulin resistance at ambient temperature.

Pharmacologic inhibition of IP6K enhances thermogenic EE and blocks the progression of HFD-induced obesity. Thus far, our results demonstrate that adipocyte-specific Ip6k1 deletion enhances thermogenic EE and global insulin sensitivity, which protects mice from HFD-induced weight gain and insulin resistance. Moreover, the lean phenotype of AdKOs resembles global Ip6k1 KO mice (58). Therefore, we monitored impacts of pharmacologic inhibition of the inositol pyrophosphate pathway on progression of dietinduced obesity (DIO) in C57BL/6 mice (Figure 4A). Mildly obese (4 weeks HFD-fed) mice were injected with vehicle or TNP for 7 weeks (Figure 4, A and B). Total body weight and weight gain are less in TNP-treated mice (Figure 4B and Supplemental Fig- 
A

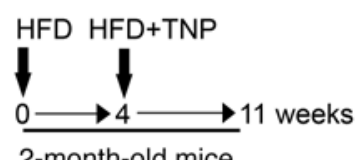

2-month-old mice
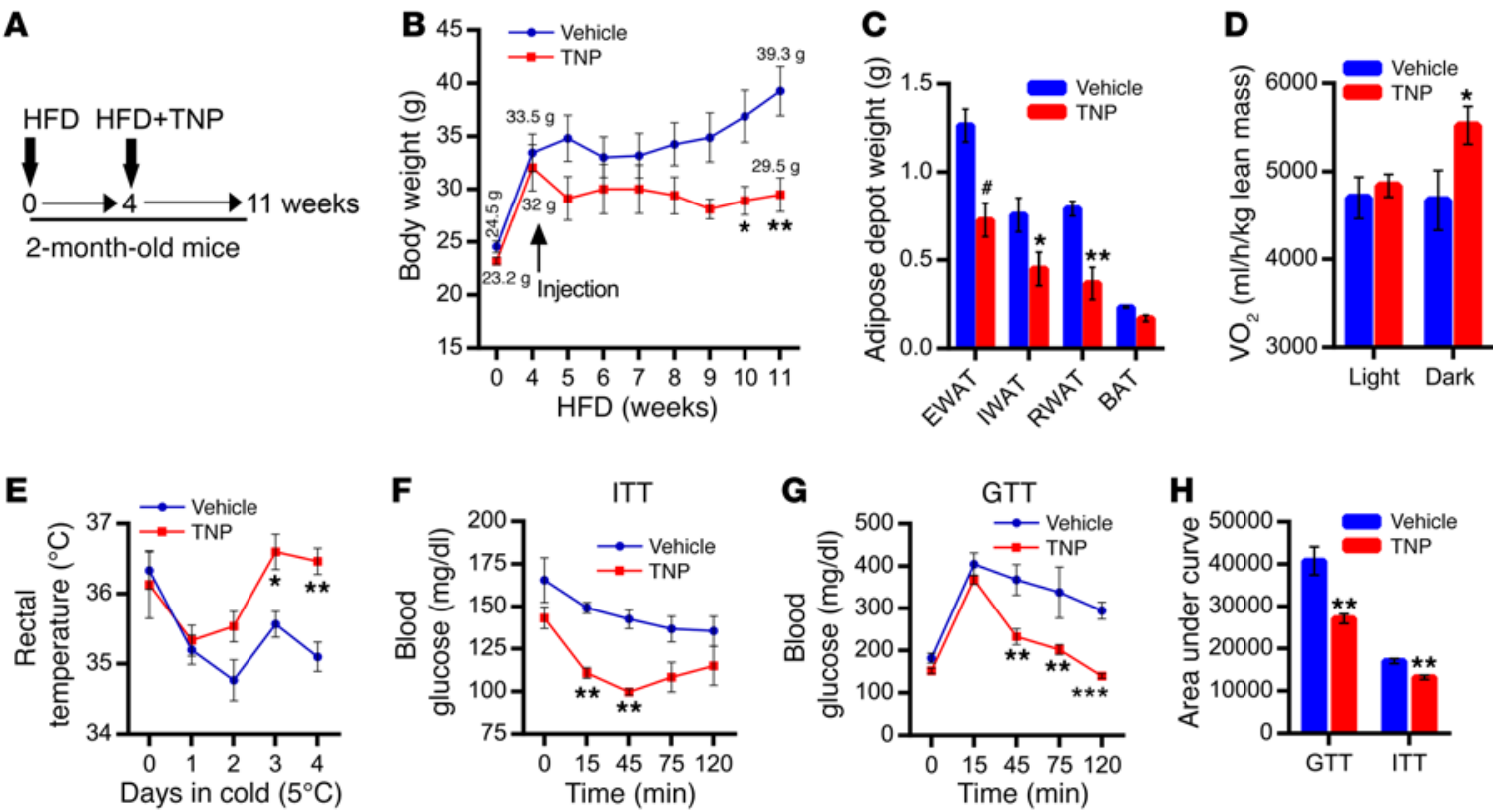

Figure 4. Pharmacologic inhibition of IP6K enhances thermogenic EE and blocks the progression of HFD-induced obesity. (A) Study design: Effects of TNP injection on body weight and metabolic parameters of HFD-fed mice. Mice were fed a HFD for 1 month. Thereafter, TNP (10 mg/kg body weight, daily; i.p.) was injected for 7 weeks ( $n=5$ mice per group). (B) Average initial body weights of 8-week-old vehicle and TNP groups (before HFD feeding or injection) are $24.5 \mathrm{~g}$ and $23.2 \mathrm{~g}$, respectively. After 4 weeks of HFD feeding, average body weights of vehicle and TNP groups are 33.5 and 32.0 g, respectively. Following 7 weeks of injection, body weights of vehicle and TNP mice were $39.3 \mathrm{~g}$ and $29.5 \mathrm{~g}$, respectively ( $n=5$ mice per group; 2 -way ANOVA). (C) Weights of diverse adipose tissue depots are less in TNP-treated mice ( $n=5$ mice per group; $t$ test). (D) TNP-treated mice display higher V ${ }_{2}$ consumption especially under dark conditions ( $n=5$ mice per group; $t$ test). (E) TNP-treated mice are protected against cold-induced decline in body temperature ( $n=3$ mice per group; 2 -way ANOVA). (F and G) TNP treatment improves glucose disposals induced by glucose (GTT) or insulin (ITT) in HFD-fed mice ( $n=5$ mice per group; 2 -way ANOVA). (H) AUC analyses of F and $\mathbf{G}\left(n=5\right.$ mice per group; $t$ test). Data in all panels are expressed as mean \pm SEM. ${ }^{*} P<0.05,{ }^{* *} P<0.01,{ }^{*} P<0.001$.

ure 4A). During the treatment period, vehicle mice gained $5.8 \mathrm{~g}$ while TNP mice lost $2.5 \mathrm{~g}$ body weight (Supplemental Figure $4 \mathrm{~B}$ ). Accordingly, TNP reduces weight of diverse adipose tissue depots (Figure 4C). TNP mice also display enhanced $\mathrm{VO}_{2}$ consumption, especially during dark conditions (Figure 4D), whereas energy intake is unaltered (data not shown). Total activity is similar in vehicle- and TNP-treated mice (Supplemental Figure 4C). TNP enhances thermogenic EE, as it protects these mice from coldinduced decline in body temperature (Figure 4E). Moreover, TNP improves glucose disposals following exogenous insulin (ITT) and glucose (GTT) injections (Figure 4, F-H).

Adipocyte-specific Ip6k1 deletion enhances browning. Next, we determined the mechanism by which IP6K1 regulates thermogenic EE. First, we monitored browning in Ip6k1-deleted adipose tissue. Chronic cold exposure dramatically induces adipose tissue remodeling, which resembles browning, especially in AdKO IWAT and RWAT (Figure 5A). PGC1- $\alpha$ and UCP1 expression levels are higher in AdKOs at $23^{\circ} \mathrm{C}$, and further enhanced following chronic cold exposure (Figure 5B). Accordingly, PGC1- $\alpha$ and UCP1 protein levels are also elevated in IWAT and RWAT but not in BAT of AdKOs (Figure 5, C and D). Other components of the thermogenic/EE machinery such as Cidea, the transcriptional coregulator $\operatorname{Prdm16}$, the transcription factors $\operatorname{PPAR} \alpha / \delta$, and the mitochondrial markers $C p t 1$ and COX 2 are also elevated in chronic-cold-exposed AdKO IWAT (Figure 5E).

Thereafter, we determined whether $I p 6 k 1$ deletion enhances browning in a cell-autonomous manner. Stromal vascular frac- tions (SVFs) isolated from IWAT of LoxPs and AdKOs were differentiated to beige adipocytes. AdKO SVFs display higher beige adipogenesis (Figure $5 \mathrm{~F}$ ), which is further evidenced by enhanced expression of the thermogenic/EE machinery (Figure 5G). Accordingly, mitochondrial oxygen consumption rate (OCR) and proton leak are higher in AdKO beige adipocytes (Figure 5, H and I). Glycolysis is unaltered in AdKO adipocytes (Supplemental Figure 5A). In contrast, browning/thermogenic marker expression levels are largely unaltered in AdKO BAT adipocytes (Supplemental Figure 5B). Thus, Ip $6 k 1$ deletion enhances browning of WAT.

IP6K1 reduces AMPK-mediated adipocyte browning. How might Ip6k1 deletion enhance browning and thermogenesis? AdKO IWAT SVFs display enhanced browning in a cell-autonomous manner (Figure 5, F and G). Moreover, global PKA signaling, tyrosine hydroxylase protein level, and $\beta$-AR (Adrb3) expression level are unaltered in cold-exposed AdKO IWAT (Supplemental Figure $6, \mathrm{~A}$ and $\mathrm{B}$ ). These results suggest that hyperactivation of the sympathetic/ $\beta$-AR pathway in AdKOs is unlikely. Conversely, Akt stimulatory phosphorylation is higher in chronic-cold-induced AdKO IWAT (Supplemental Figure 6, C and D). Activation of the PI3K/Akt pathway enhances browning, UCP1 expression, and glucose uptake (74-76). Moreover, ADIPOQ (which is higher in AdKOs) enhances cold-induced browning of subcutaneous adipose tissue via Akt-mediated proliferation of M2 macrophages (76). Adipoq expression is higher in the chronic-cold-exposed AdKO IWAT, yet the macrophage population is unaltered in this condition (Supplemental Figure 6E). Thus, although the ADIPOQ/Akt 
A

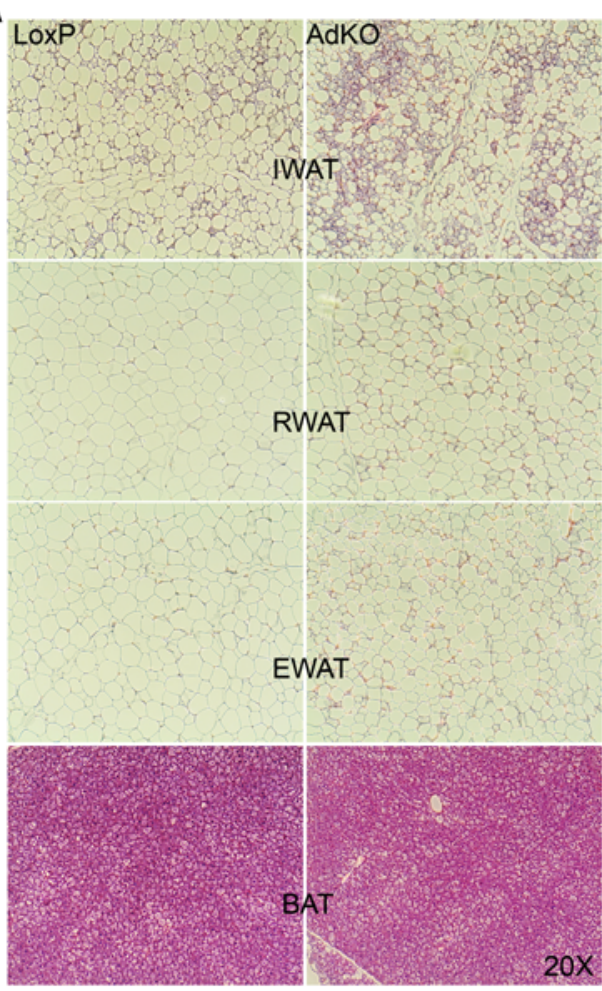

Chow: cold, 6 days (H\&E)
B

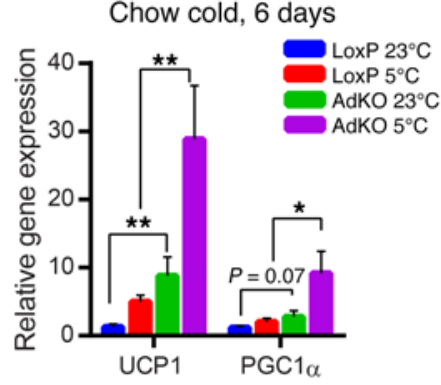

D

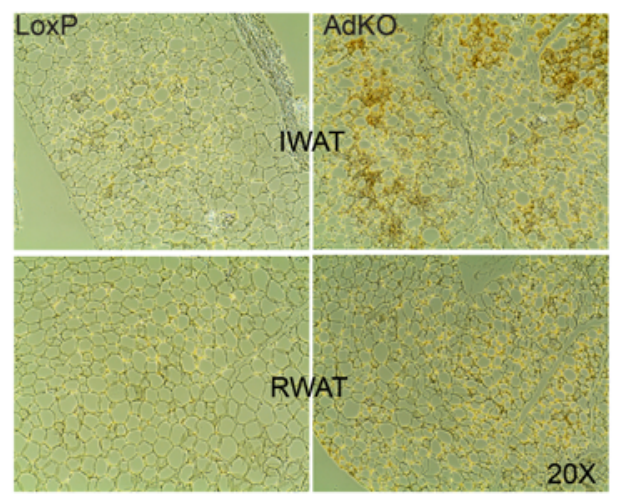

Chow: cold, 6 days (UCP1 staining)
C Chow cold, 6 days

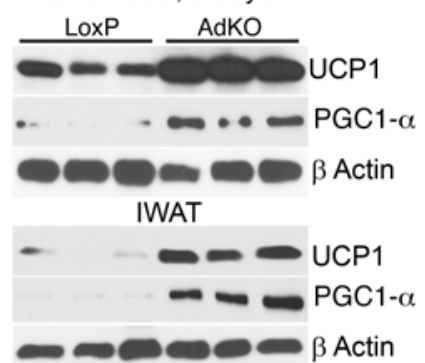

RWAT

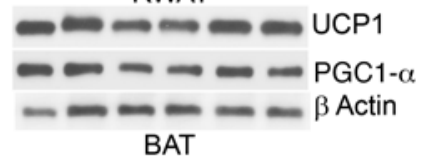

E

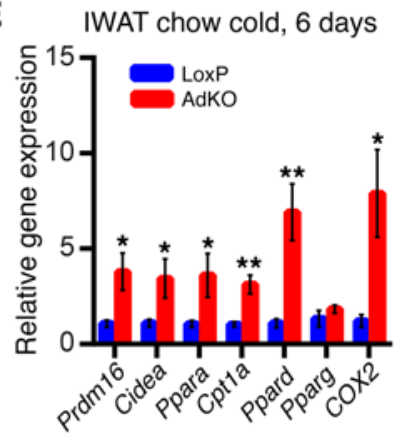

$\mathbf{F}$

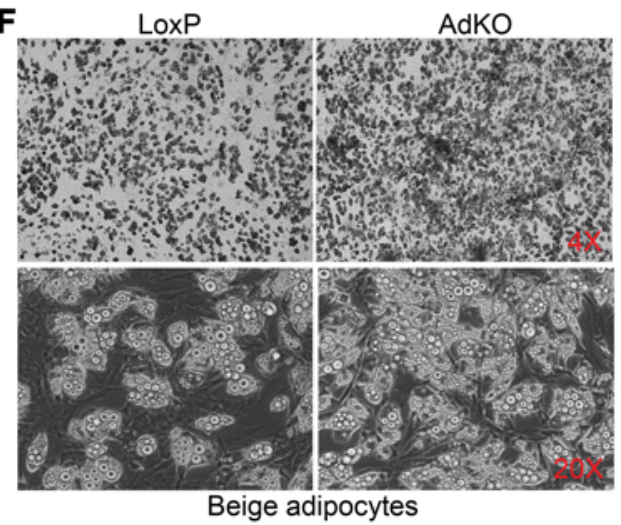

G

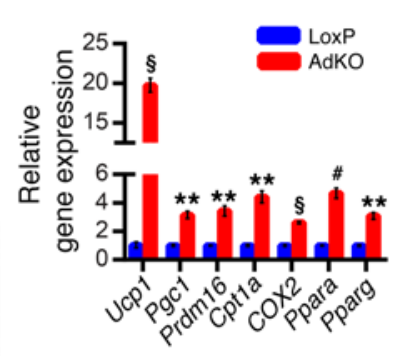

H

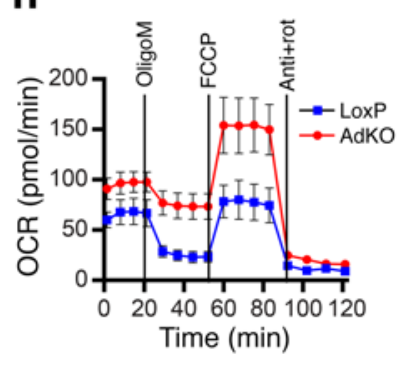

I

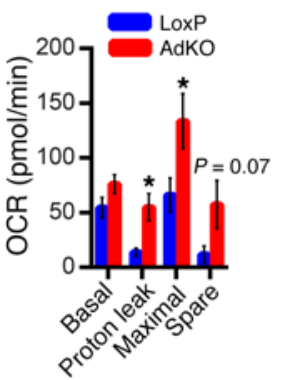

Figure 5. Adipocyte-specific Ip6k1 deletion enhances browning. (A) Chronic cold enhances adipose tissue remodeling that resembles browning in CDAdKOs (image represents data from 3 individual mice per group). (B) Ucp1 and Pgc1 expression levels are higher in AdKOs at $23^{\circ} \mathrm{C}$ and are further enhanced by chronic cold exposure ( $n=6$ mice per group; 1-way ANOVA). (C) Chronic cold enhances PGC1- $\alpha$ and UCP1 protein levels in CD-AdKO IWAT and RWAT ( $n=3$ mice per group). (D) Immunohistochemistry reveals enhanced UCP1 protein levels in chronic-cold-exposed CD-AdKO IWAT and RWAT (images represent results obtained from $n=3$ mice per group). (E) Browning and thermogenic and mitochondrial EE machinery are upregulated in chronic-cold-exposed CD-AdKOs ( $n=6$ mice per group; $t$ test). (F) SVFs ( $n=6$ mice per preparation) isolated from AdKO IWAT display enhanced beige adipogenesis. Images represent results obtained from at least 3 independent experiments. (G) Beige and mitochondrial activity markers are upregulated in AdKO IWAT beige adipocytes ( $n=6$ mice per preparation; triplicate samples). (H) OCR is higher in AdKO IWAT beige adipocytes ( $n=6$ mice per preparation; 10 replicates). OligoM, oligomycin; Anti, antimycin A; rot, rotenone. (I) Quantification of $\mathbf{H}$ reveals that average proton leak and maximal respiratory capacity are significantly higher in AdKO IWAT beige adipocytes. Data in all panels are expressed as mean \pm SEM. ${ }^{*} P<0.05,{ }^{* *} P<0.01,{ }^{*} P<0.001,{ }^{\circledR} P<0.0001$.

axis is more active in cold-exposed AdKOs, it may not have exerted its influence on macrophages at least under the conditions tested.

Further analyses reveal that the stimulatory phosphorylation (T172) of AMPK is slightly higher in chronic-cold-exposed AdKOs (Supplemental Figure 6F). AMPK is sensitive to acute and subtle changes in energy and stress. Thus, we presumed that chronic cold exposure may have saturated AMPK activity. Indeed, WT mice display substantially enhanced AMPK stimulatory phosphorylation following acute cold exposure (Figure 6A). Therefore, we tested AMPK activity in acute-cold-exposed LoxPs and AdKOs. Acutecold-induced AMPK stimulatory phosphorylation is substantially higher in AdKOs (Figure 6B and Supplemental Figure 6G). AMPK 
A

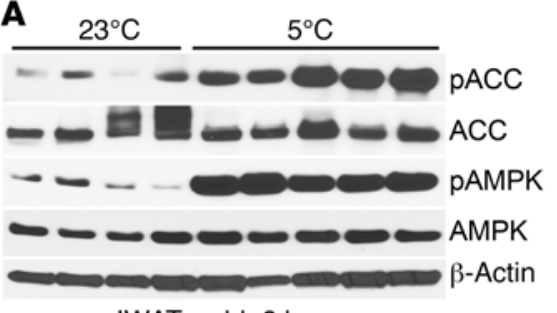

IWAT: cold, $6 \mathrm{~h}$

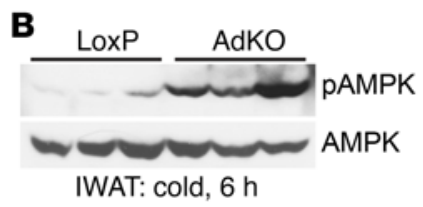

IWAT: cold, $6 \mathrm{~h}$

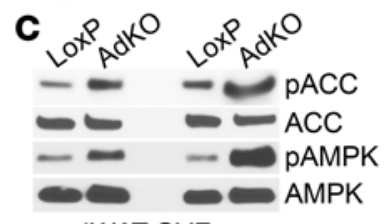

IWAT-SVF:

beige adipocytes

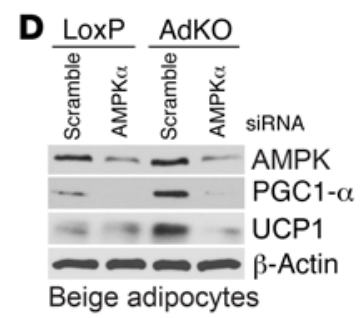

I

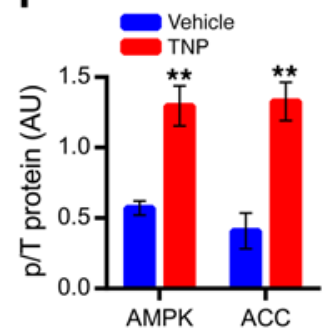

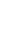

E

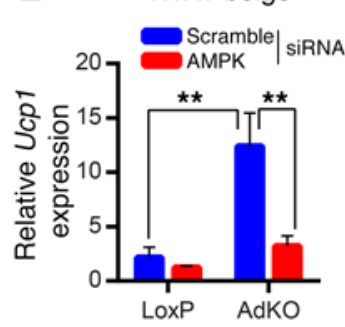

$\mathbf{F}$

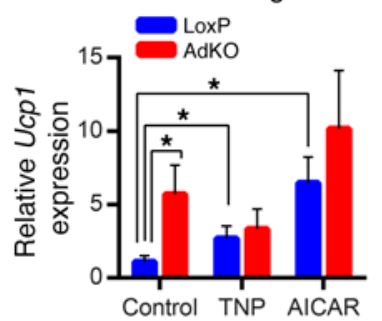

G

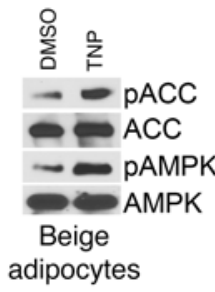

H

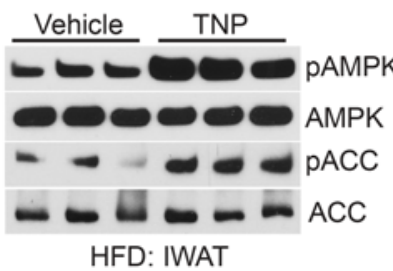

Figure 6. IP6K1 reduces AMPK-mediated adipocyte browning. (A) AMPK stimulatory phosphorylation (T172) and its activity are enhanced in WT mice following acute cold exposure ( $n=4-5$ mice per group). (B) AMPK phosphorylation is dramatically augmented in acute-cold-exposed AdKOs ( $n=3$ mice per group). (C) AMPK activity is higher in 2 separate preparations ( $n=6$ mice per preparation) of AdKO beige adipocytes differentiated from IWAT SVF in vitro. (D) siRNA-mediated AMPK $\alpha$ depletion substantially reduces AMPK $\alpha$ protein level in LoxP and AdKO beige adipocytes. Moreover, PGC1- $\alpha$ and UCP1 protein levels, which are higher in AdKO adipocytes, are reduced following AMPK $\alpha$ depletion. Data represent results obtained from 2 independent experiments. (E) Ucp1 mRNA expression is slightly reduced by AMPK $\alpha$ siRNA treatment in LoxP mice. Conversely, Ucp1 expression, which is significantly higher in AdKO adipocytes compared with LoxPs, is substantially reduced following AMPK $\alpha$ depletion. Data represent results obtained from 5 experimental replicates. (F) AdKO beige adipocytes express higher levels of Ucp1 compared with LoxP adipocytes under basal conditions (control). TNP (5 $\mu$ M, 16 hours) substantially enhances Ucp1 expression in LoxP adipocytes, whereas it does not influence Ucp1 in AdKO adipocytes. The AMPK activator AICAR enhances Ucp1 expression to a higher extent in LoxP adipocytes. Data represent results obtained from 5 experimental replicates. (G) TNP (5 $\mu$ M) enhances AMPK phosphorylation and activity in beige adipocytes differentiated in vitro from isolated from WT IWAT SVF. Immunoblot represents results from at least 3 independent experiments. (H and I) A single dose of TNP (20 mg/ $\mathrm{kg}$ body weight; i.p.) enhances AMPK phosphorylation and activity on ACC in the IWAT of HFD-fed mice ( $n=3$ mice per group; $t$ test). Data in all panels are expressed as mean $\pm S E M .{ }^{*} P<0.05,{ }^{* *} P<0.01$.

phosphorylation is also higher in 2 separate preparations of beige adipocytes differentiated from IWAT SVFs of AdKOs (Figure 6C). Moreover, Ip $6 k 1$ deletion-mediated enhancement in adipose tissue browning is modulated by AMPK, as AMPK $\alpha$ depletion reduces PGC1- $\alpha$ and UCP1 levels in AdKO adipocytes (Figure 6, D and E).

Next, we tested whether pharmacologic inhibition of IP6Ks or activation of AMPK stimulates UCP1 expression in beige adipocytes. TNP (5 $\mu \mathrm{M}, 16$ hours) substantially enhances UCP1 expression in LoxP adipocytes (Figure 6F). Conversely, TNP does not further stimulate UCP1 expression in AdKO adipocytes (Figure 6F). The AMPK activator AICAR significantly enhances UCP1 expression in LoxP adipocytes albeit its effects on AdKO adipocytes are minimal (Figure 6F). Moreover, TNP enhances AMPK phosphorylation and activity in LoxP beige adipocytes (Figure 6G). Therefore, we monitored whether TNP enhances AMPK activity in the adipose tissue of HFD-fed mice. Indeed, a single dose of TNP substantially enhances AMPK phosphorylation and its activity in the IWAT of HFD-fed mice (Figure 6, $\mathrm{H}$ and I). Thus, IP6K1 regulates adipose tissue browning via modulation of the AMPK pathway.

IP6 and IP6K1 differentially regulate AMPK stimulatory phosphorylation. Next, we determined the mechanism by which IP6K1 regulates AMPK activity and signaling. Overexpression of Ip $6 k 1$ in HEK293 cells reduces phosphorylation and activity of AMPK on its target ACC (Figure 7A). Thereafter, we monitored effects of various inositol phosphates and pyrophosphate on LKB1-mediated AMPK phosphorylation in a purified in vitro system. We used a commercially available unphosphorylated (UP) AMPK $\alpha 2 / \beta 1 / \gamma 1$ complex as the LKB1 substrate (Supplemental Figure 7A). LKB1 requires coactivators MO25 and STRAD $\alpha$ for its full activity, and thus, in the absence of these coactivators, LKB1 is partly active (36). We used both LKB1-only (without coactivators) and LKB1complex (with coactivators) on AMPK $\alpha 2 / \beta 1 / \gamma 1$ phosphorylation. First, we used LKB1-only, which phosphorylates UP-AMPK (Supplemental Figure 7, B and C). Next, we added increasing concentrations of IP6 and 5-IP7 in the assay. IP6 but not 5-IP7 potently stimulates LKB1-only-mediated UP-AMPK phosphorylation (Figure 7B). IP6 displays an $\mathrm{EC}_{50}$ of about $150 \mathrm{nM}$ (Figure 7C and Supplemental Figure 7D). Among various inositol polyphosphates, IP6 is the most potent in the assay, followed by IP5, whereas others are less effective or are ineffective (IP6 $>$ IP5 $>$ IP4 $=$ IP5 ${ }^{*}>$ IP3 $>$ 5-IP7) (Figure 7D). The products (5-IP7 and IP5*) of IP6K1 do not display any stimulatory effect on AMPK phosphorylation (Figure 7D). Thus, it is conceivable that in the presence of IP6K1, IP6 is converted to either 5-IP7 or IP5*, which neutralizes IP6-mediated AMPK activation. To test this, we used purified catalytically active and inactive versions of recombinant human IP6K1 (49). Catalytically active IP6K1 potently decreases IP6's stimulatory action, whereas the inactive IP6K1 is largely ineffective (Figure 7E). 5-IP7, at physiological concentration, also reverses IP6's stimulatory 
A

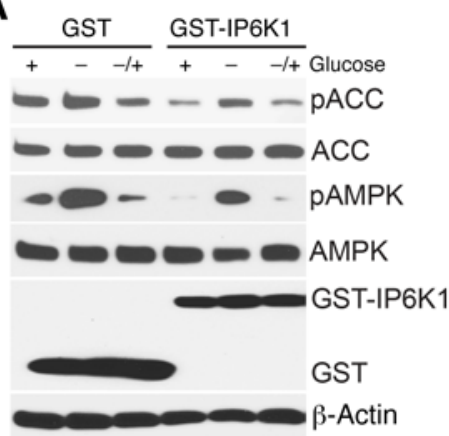

D

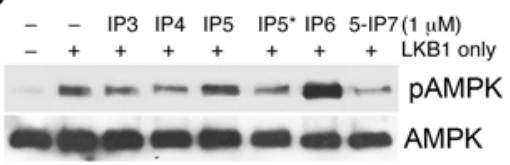

LKB1-only activity on AMPK $\alpha 2 / \beta 1 / \gamma 1$
B

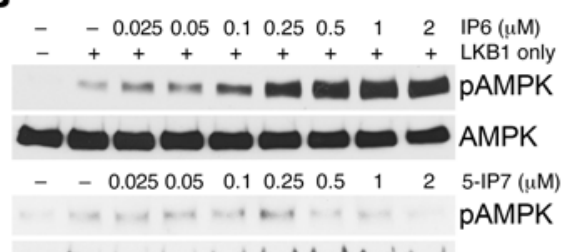

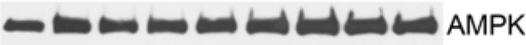

LKB1-only activity on AMPK $\alpha 2 / \beta 1 / \gamma 1$

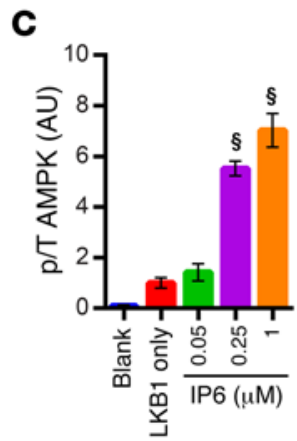

\section{E}

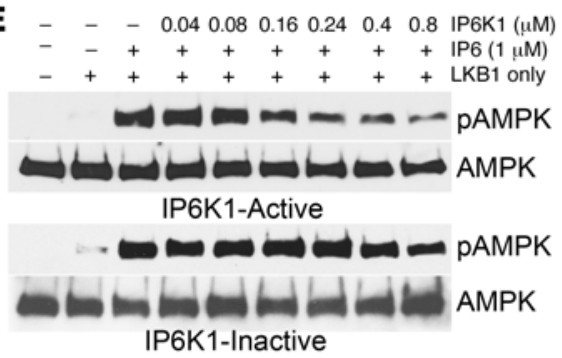

LKB1-only activity on AMPK $\alpha 2 / \beta 1 / \gamma 1$

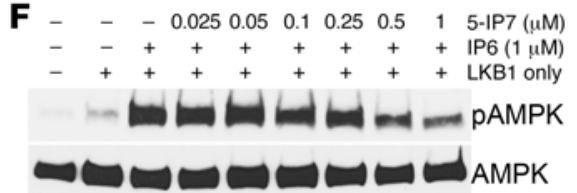

LKB1-only activity on AMPK $\alpha 2 / \beta 1 / \gamma 1$

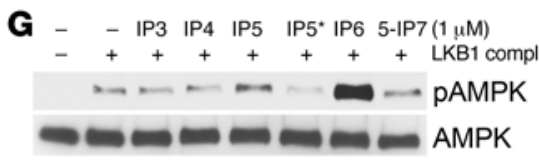

LKB1 compl activity on AMPK $\alpha 2 / \beta 1 / \gamma 1$

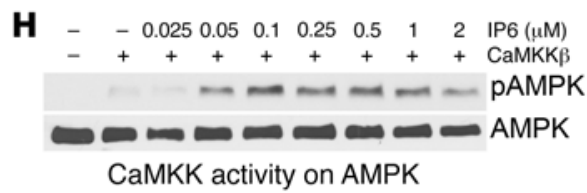

CaMKK activity on AMPK

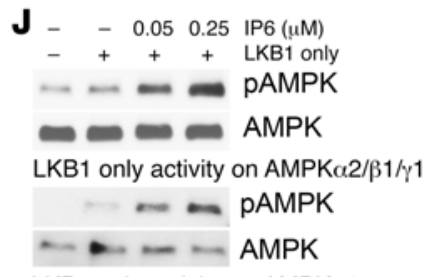

LKB1 only activity on AMPK $\alpha 2$

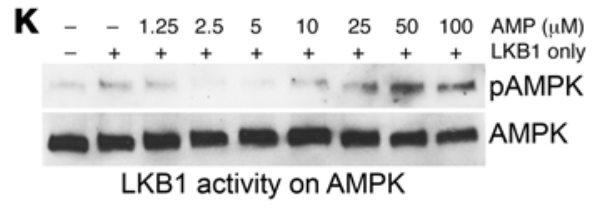

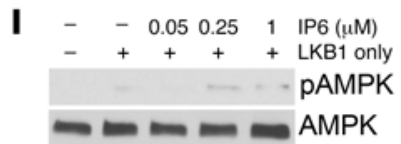

LKB1 only activity on heat-denatured AMPK $\alpha 2 / \beta 1 / \gamma 1$

$\mathbf{L}$

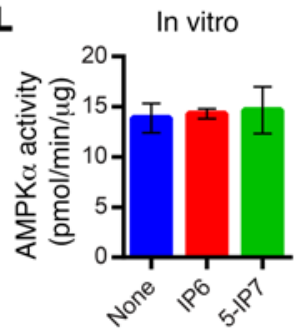

Figure 7. IP6 and IP6K1 differentially regulate AMPK stimulatory phosphorylation. (A) Immunoblot demonstrates that overexpression of CST-IP6K1 diminishes AMPK and ACC phosphorylation levels in HEK293 cells under basal (+), glucose-deprived (-), and glucose-reintroduced (-/+) conditions. Data represent results from at least 3 independent experiments. (B) IP6, at increasing concentrations, stimulates AMPK $\alpha 2 / \beta 1 / \gamma 1$ (AMPK $\alpha$ T172) phosphorylation mediated by LKB1-only (120 ng/reaction). Data represent results from at least 3 independent experiments. (C) Quantification of B reveals that IP6 leads to 6- to 7-fold enhancements in LKB1-mediated AMPK phosphorylation in vitro. Data represent results from at least 3 independent experiments (1-way ANOVA). (D) Among inositol phosphates, IP6 most potently stimulates LKB1-only-mediated AMPK $\alpha 2 / \beta 1 / \gamma 1$ phosphorylation (IP6 $>$ IP5 $>$ IP4 = IP5* $>$ IP3 $>$ 5-IP7). IP5 displays partial stimulatory effect. IP6K1 products IP5* and 5-IP7 are ineffective. Data represent results from duplicate experiments. (E) IP6K1 inhibits IP6-mediated AMPK $\alpha 2 / \beta 11$ phosphorylation by LKB1-only, whereas the inactive IP6K1 mutant is much less efficient. Data represent results from 3 independent experiments. (F) 5-IP7 reverses IP6-mediated AMPK $\alpha 2 / \beta 1 / \gamma 1$ phosphorylation by LKB1-only. Data represent results obtained from 3 independent experiments. (G) IP6 also enhances LKB1-complex-mediated AMPK $\alpha 2 / \beta 11$ phosphorylation. Other inositol polyphosphates are ineffective. Data represent results obtained from duplicate experiments. (H) IP6 enhances CaMKK $\beta$-mediated AMPK $\alpha 21 / \gamma 1$ phosphorylation. Data represent results obtained from duplicate experiments. (I) IP6 does not enhance LKB1-mediated phosphorylation of heat-denatured AMPK $\alpha 2 / \beta 1 / \gamma 1$. Data represent results obtained from 3 independent experiments. (J) IP6-mediated enhancement of AMPK phosphorylation is largely similar when AMPK $\alpha$ is used alone or in a complex with AMPK $\alpha 2 / \beta 1 / \gamma 1$. Data represent results obtained from 3 independent experiments. (K) AMP stimulates LKB1-mediated AMPK $\alpha 2 / \beta 11$ phosphorylation at 50- to 100- $\mu \mathrm{M}$ concentrations. Data represent results obtained from duplicate experiments. (L) IP6 or 5-IP7 does not influence active AMPK's activity on its target SAMStide ( $n=3 ; 1$-way ANOVA). Data are expressed as mean \pm SEM. ${ }^{\S} P<0.0001$.

effect (Figure 7F). We presume that 5-IP7 (1-5 $\mu \mathrm{M}$ in cells) is less likely to inhibit IP6-mediated activation, as IP6 is more potent in the assay, and is abundant $(\sim 50 \mu \mathrm{M})$ in cells (45). Rather, it is conceivable that IP6K1 interferes with IP6's action on AMPK via removal of IP6 by converting it to 5-IP7 or IP5*. Endogenous IP6K1 and AMPK $\alpha$ interact, which supports this possibility (Supplemental Figure 7E). Overexpression of glutathione $S$-transferase (GST)IP6K1 deletion mutants reveals that the IP6K1 146-205 fragment 
A

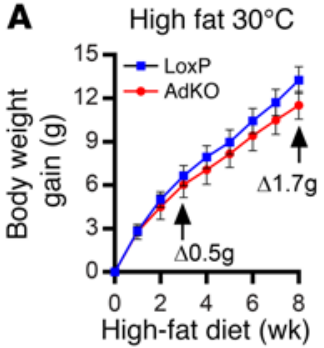

B

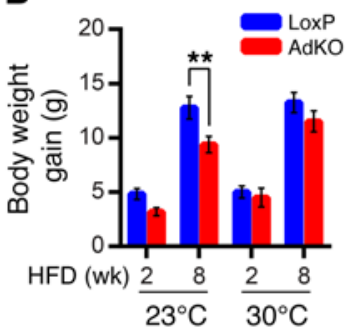

C

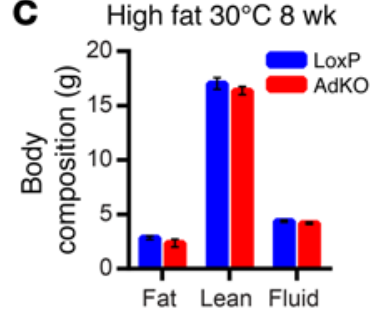

D High fat $30^{\circ} \mathrm{C} 8 \mathrm{wk}$

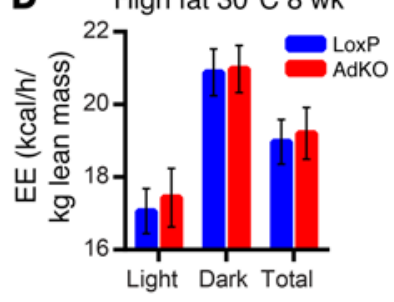

$\mathbf{E}$

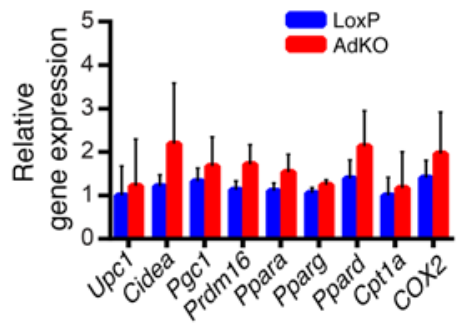

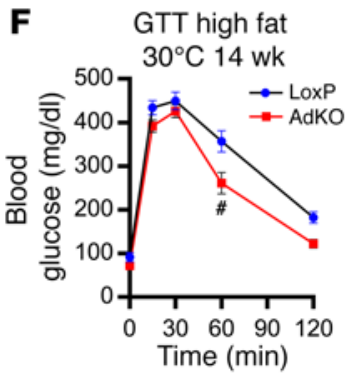

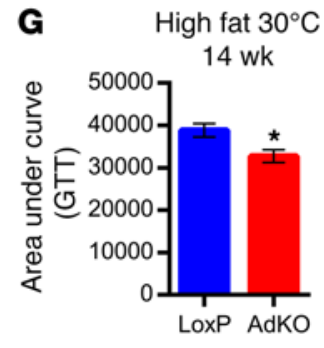

Figure 8. Thermoneutrally placed HFD-AdKO mice do not display leanness but exhibit insulin sensitivity. (A) AdKOs, when fed a HFD at thermoneutral $\left(30^{\circ} \mathrm{C}\right.$ ) temperature, gain body weight at a similar rate compared with LoxPs ( $n=6$ mice per group; 2 -way ANOVA). (B) AdKOs at $23^{\circ} \mathrm{C}(8 \mathrm{weeks}$ of HFD) display a reduced weight gain compared with LoxPs, whereas AdKOs at $30^{\circ} \mathrm{C}$ gain similar body weight compared with LoxPs ( $n=6$ mice per group; $t$ test). (C) Total fat, lean, and fluid masses are similar in LoxPs and AdKOs after 8 weeks of HFD feeding at $30^{\circ} \mathrm{C}(n=6$ mice per group; $t$ test). (D) EE is similar in AdKOs and LoxPs at $30^{\circ} \mathrm{C}$ ( 8 weeks of HFD) ( $n=6$ mice per group; $t$ test). (E) Adipose tissue browning and mitochondrial markers are similar in HFD-fed LoxPs and AdKOs, after 14 weeks of HFD at thermoneutrality ( $n=6$ mice per group; $t$ test). (F) After 14 weeks of HFD at $30^{\circ} \mathrm{C}$, AdKOs display improved GTT compared with LoxPs ( $n=6$ per group; 2-way ANOVA). (G) AUC analyses reveal that AdKOs, after 14 weeks of HFD at $30^{\circ} \mathrm{C}$, efficiently dispose glucose compared with LoxPs ( $n=6$ mice per group; $t$ test). (H) Akt stimulatory (S473) phosphorylation is 4 - to 5 -fold higher in AdkO liver and EWAT after 14 weeks of HFD feeding at $30^{\circ} \mathrm{C}\left(n=4\right.$ mice per group). Data in all panels are expressed as mean $\pm \mathrm{SEM}$. ${ }^{*} P<0.05,{ }^{* *} P<0.01,{ }^{*} P<0.001$.

binds with AMPK $\alpha$ (Supplemental Figure 7F). Moreover, the IP6K inhibitor TNP, which enhances intracellular IP6 level $(58,67)$ and AMPK activity in beige adipocytes, adipose tissue (Figure 6, G and $\mathrm{H}$ ), and 3T3L1 cells (Supplemental Figure 7G), disrupts IP6K1AMPK $\alpha$ interaction (Supplemental Figure $7 \mathrm{H}$ ).

Does IP6 regulate LKB1 or other upstream kinases that phosphorylate AMPK? IP6 stimulates partially active (without the cofactors MO25 and STRAD $\alpha$ ) LKB1 mediated AMPK phosphorylation. It is conceivable that, like its cofactors, IP6 alters LKB1 conformation and thus activates the enzyme. In such a case, IP6 should be less effective or be ineffective on AMPK phosphorylation mediated by active LKB1 (with cofactors). To test this, we used active LKB1 (LKB1-complex), which, as expected, phosphorylates AMPK more robustly than LKB1-only (Supplemental Figure 7I). However, IP6 still stimulates LKB1-complex-mediated AMPK phosphorylation (Supplemental Figure 7, J and K). Like the LKB1-only assay, inositol polyphosphates other than IP6 are largely ineffective (Figure 7G). IP6K1 also inhibits IP6's effects on LKB1-complex-mediated AMPK phosphorylation to a similar extent compared with its effects on the LKB1-only assay (Supplemental Figure 7L). Moreover, IP6 stimulates CaMKK $\beta$-mediated AMPK phosphorylation (Figure $7 \mathrm{H}$ ). These results suggest that IP6 may not influence upstream kinases of AMPK. Conversely, IP6 fails to activate LKB1-only-mediated AMPK phosphorylation when AMPK $\alpha 2 / \beta 1 / \gamma 1$ conformation is disrupted by heat denaturation (Figure 7I). IP6 activates LKB1-only-mediated phosphorylation of the AMPK $\alpha 2 / \beta 1 / \gamma 1$ complex and AMPK $\alpha$ alone to a similar extent, which indicates that IP6 may influence AMPK $\alpha$ conforma- tion (Figure 7J). AMP, the known AMPK activator, is less effective than IP6 in stimulating LKB1-only-mediated AMPK phosphorylation (Figure 7K). Conversely, IP6 and 5-IP7, unlike AMP, do not stimulate phosphorylated/active AMPK's catalytic activity on SAMStide (Figure 7L and Supplemental Figure 7M). Unlike AMP, IP6 and IP6K1 do not directly influence active AMPK's activity on its targets. Thus, IP6 enhances AMPK phosphorylation, whereas IP6K1's interference neutralizes IP6's stimulatory action (a) by IP6K1's binding to its bona fide substrate IP6 and/or (b) by IP6K1mediated conversion of IP6 to 5-IP7 or IP5*.

Thermoneutrally placed HFD-AdKO mice do not display leanness, though they exhibit insulin sensitivity. If enhanced thermogenic EE reduces fat accumulation in AdKO mice, placing them at thermoneutral $\left(30^{\circ} \mathrm{C}\right)$ temperature would, at least in part, impair their lean phenotype. Indeed, in this condition, HFD-fed LoxPs and AdKOs display largely similar body weight gain after 8 weeks (Figure 8A). $\mathrm{HFD}$-induced body weight gain in $\mathrm{AdKOs}$ at $23^{\circ} \mathrm{C}$ is substantially less than that in LoxPs, whereas it is similar at $30^{\circ} \mathrm{C}$ (Figure $8 \mathrm{~B}$ ). Accordingly, thermoneutral HFD-AdKOs do not display any alterations in body composition (Figure 8C). EE, which is higher in AdKOs at $23^{\circ} \mathrm{C}$ after 8 weeks of HFD (Supplemental Figure 2J), is unaltered at $30^{\circ} \mathrm{C}$ (Figure $8 \mathrm{D}$ ). RER and total activity profiles are also unchanged (Supplemental Figure 8, A and B). Glucose disposal rate is similar in HFD-fed LoxPs and AdKOs in this condition (Supplemental Figure 8, C and D).

To monitor whether chronic exposure to HFD at thermoneutrality alters body weight, we continued the same experiment for 14 weeks. Even after 14 weeks of HFD feeding at $30^{\circ} \mathrm{C}$, AdKOs gain 

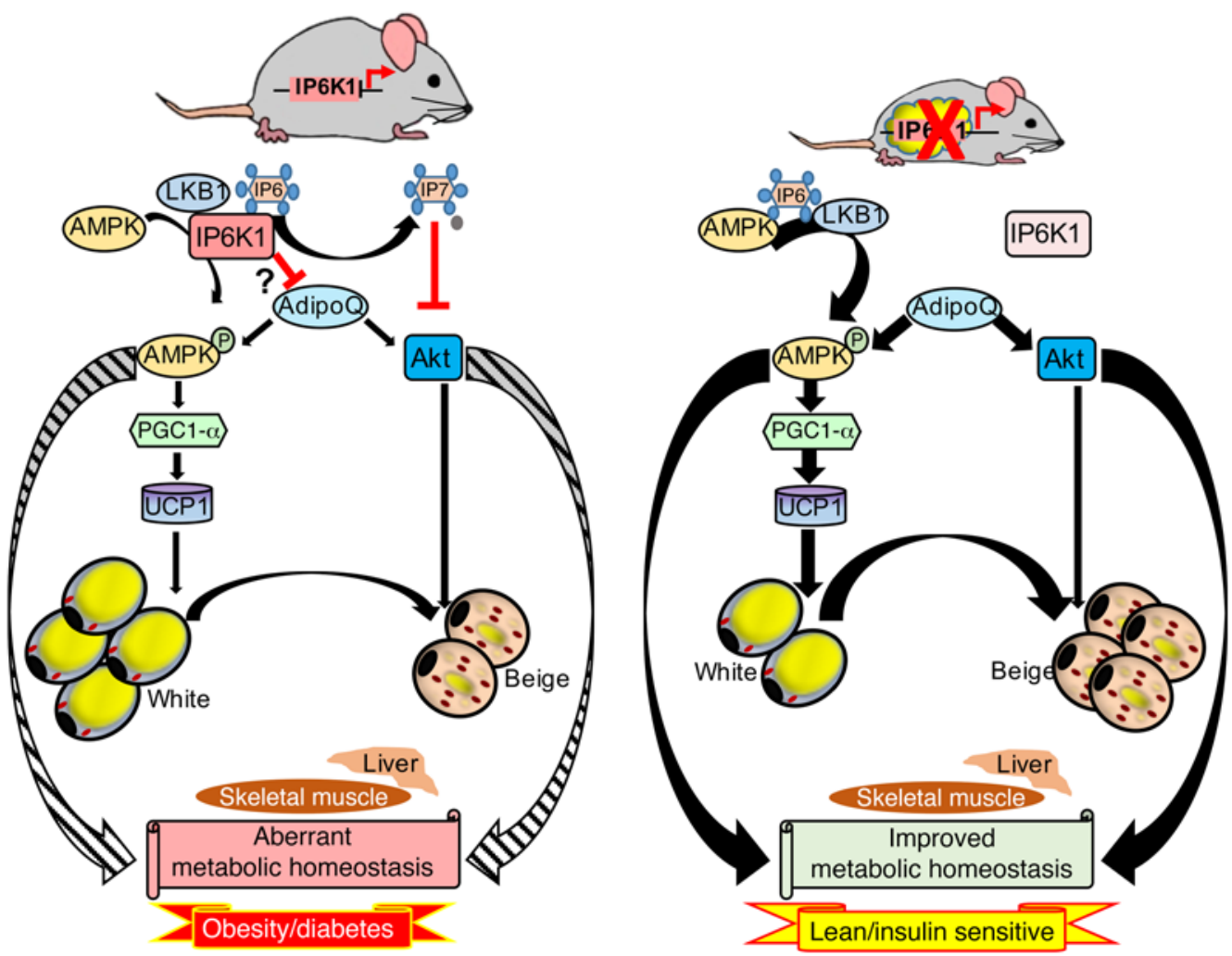

Figure 9. IP6K1 regulation of adipose tissue metabolism modulates whole-body energy homeostasis. Left panel: Adipocyte-specific IP6K1 regulates energy metabolism by inhibiting the AMPK pathway via a novel mechanism. IP6 enhances upstream kinase-mediated AMPK stimulatory phosphorylation. IP6K1, on the other hand, inhibits AMPK phosphorylation by generating 5-IP7 from IP6. AMPK activates the transcriptional co-activator PGC1 $\alpha$, which is a major regulator of adipose tissue browning and UCP1 mediated thermogenic EE. Moreover, IP6K1 generated 5-IP7 inhibits Akt. IP6K1 also reduces serum levels of ADIPOQ, which indirectly influences these pathways. Thus, adipocyte-specific IP6K1 promotes DIO and insulin resistance. Right panel: In AdKO mice, Akt, AMPK, and ADIPOQ signaling is upregulated, which leads to thermogenic EE mediated by adipose tissue browning and to global insulin sensitivity. As a result, AdKO mice are protected against HFD-induced weight gain and insulin resistance. Thus, adipocyte-specific IP6K1 is a major regulator of global energy metabolism. Striped and bold encircling arrows indicate reduced and increased effects of AMPK and Akt on tissue metabolism, in the presence and absence of IP6K1, respectively. Thin and thick straight arrows indicate less and more active pathways, respectively.

largely similar body weight compared with LoxPs (Supplemental Figure 8E). Accordingly, adipose tissue browning and mitochondrial activity markers are similar in LoxPs and AdKOs under this condition (Figure $8 \mathrm{E}$ ), which further suggests that leanness in AdKOs is due to increased thermogenesis. However, at $30^{\circ} \mathrm{C}$, AdKOs fed HFD for 14 weeks dispose glucose more efficiently than LoxPs (Figure 8, F and G). Akt stimulatory phosphorylation is also higher in AdKO adipose tissue and liver under this condition (Figure 8H and Supplemental Figure 8F). Thus, 14 weeks of thermoneutrality abolishes leanness/browning in HFD-AdKOs, whereas insulin sensitivity is retained. Although it is not entirely clear at this point, we presume that IP6K1-mediated distinct regulation of browning and insulin sensitivity partly uncouples the lean and insulin-sensitive phenotype at thermoneutral temperature.

\section{Discussion}

By generating an adipocyte-specific mouse model (AdKO), we demonstrate that IP6K1 regulates global energy homeostasis via modulation of adipose tissue energy metabolism. AdKOs display enhanced AMPK-mediated browning/thermogenesis, which protects them from HFD-induced weight gain. In addition, HFD-fed AdKOs exhibit higher activity of the insulin-sensitizing protein kinase Akt in the adipose tissue. Moreover, HFD-AdKOs show higher plasma levels of ADIPOQ. As a result, the knockouts are protected against global insulin resistance (Figure 9, model). Accordingly, pharmacologic inhibition of IP6K, by the pan-inhibitor TNP, inhibits the progression of DIO and insulin resistance at ambient $\left(23^{\circ} \mathrm{C}\right)$ temperature (Figure 4$)$. An extensive recent study from our laboratory further demonstrates that TNP inhibits the initiation of DIO at $23^{\circ} \mathrm{C}(77)$. TNP promotes weight loss in DIO WT but not in Ip6k1 KO mice, which strongly indicates that it works via the IP6K1 pathway (77). Both Akt and AMPK are less active in obese and diabetic metabolic tissues $(21,78)$. TNP enhances Akt $(77)$ and AMPK activities (Figure 6H) in DIO mice. At thermoneutral condition, TNP's effect on body weight of WT mice is significantly impaired, yet not completely abolished (77), whereas the same condition obliterated the lean phenotype of adipocyte-specific Ip6k1-deleted mice (Figure 8A). These results indicate that global inhibition of the inositol pyrophosphate pathway has greater effects on metabolism, which is conceivable, as Ip $6 k$ s are ubiquitously expressed. To obtain further insight, similar studies in the global Ip $6 \mathrm{k} 1 \mathrm{KO}$ mice are required. Thus, although, IP6K inhibition-mediated improvements in metabolic parameters are expected to have synergism with temperature variations, it may not be mandatory. 
Our study also unravels a novel mechanism by which IP6K1 regulates adipocyte browning. IP6K1's substrate IP6 stimulates LKB1-mediated AMPK phosphorylation, whereas IP6K1 and its product 5-IP7 diminish IP6's stimulatory effect. Thus, in the absence of IP6K1, AMPK activity is higher in the adipose tissue. A number of studies appreciate inositol polyphosphates as regulators of protein kinases such as BTK, CK2, and Akt $(46,58,79)$. In-depth biophysical, structural, and biochemical studies are required to further dissect the molecular mechanism by which IP6/IP6K1 regulates AMPK. 5-IP7 may also indirectly inhibit AMPK. For example, 5-IP7 promotes nuclear localization of LKB1 in cancer cells, which diminishes its activity toward the cytosolic targets (80). Cytosolic LKB1 phosphorylates AMPK (81). Accordingly, global Ip6k1 KO IWAT SVFs display higher cytosolic LKB1 (unpublished observations).

IP6/IP6K1-mediated regulation of AMPK may have broader implications, as aberration of the pathway is also observed in other diseases such as cancer, neurodegeneration, and aging. Accordingly, active research is ongoing to identify small-molecule activators of AMPK $(82,83)$. However, to our knowledge, physiological small-molecule activators of AMPK, other than AMP, are not known. Therefore, AMPK activation by the biomolecule IP6 is novel. IP6K1-AMPK $\alpha$ interaction interferes with IP6-mediated AMPK activation. Thus, disruption of the interaction seems to be critical in enhancing AMPK activity. Accordingly, TNP, which inhibits IP6K activity (64), increases IP6 levels $(58,67)$, and disrupts IP6K1-AMPK binding (Supplemental Figure 7H), dramatically enhances AMPK activity in adipocytes. Enzymes that generate and/or metabolize IP6 may also regulate AMPK. For example, the primary physiological role of inositol polyphosphate multikinase (IPMK) is to form the bulk of IP5, which is the precursor of IP6, and thus, Ipmk deletion reduces IP5 and IP6 levels $(84,85)$. IPMK-AMPK interaction is regulated by glucose availability (86). Moreover, metformin-induced AMPK stimulatory phosphorylation and activity are impaired in Ipmk-deleted MEFs, which are restored by overexpression of catalytically active IPMK (84). Thus, it is conceivable that the ratio of IP6 to 5-IP7 in a microenvironment may regulate cellular targets such as AMPK. The ratio of IP6 to 5-IP7 is altered by various intrinsic or extrinsic factors such as growth factor deprivation, altered ATP levels, chemotactic stimulation, and apoptotic stimuli $(43,45,58,59)$. In addition, various conditions that regulate expression levels of $I p 6 k 1$ (such as cold exposure) may also alter the ratio.

IP6K1-mediated regulation of EE exhibits cell/tissue specificity. For instance, global Ip6k1 KOs (58) and AdKOs display enhanced EE. Moreover, Ip6k1-deleted beige adipocytes show increased mitochondrial OCR due to proton leak, whereas glycolysis is unchanged. TNP-treated diabetic cardiomyocytes also exhibit enhanced mitochondrial biogenesis and function (66). Conversely, immortalized MEFs from global Ip $6 k 1 \mathrm{KO}$ mice are reported to display higher glycolysis whereas oxygen consumption is compromised (55). AMPK stimulates glycolysis (28); thus it is feasible that increased AMPK in certain tissues may also enhance glycolytic energy metabolism. In conclusion, improved EE following adipocyte-specific Ip $6 k 1$ deletion or IP6K inhibition indicates that the pathway may have therapeutic potential in human obesity, $\mathrm{T} 2 \mathrm{D}$, and other metabolic diseases.

\section{Methods}

Quantitative RT-PCR probes. TaqMan probes were purchased from Life Technologies.

Antibodies. Phospho-Akt (S473; catalog 4060), total Akt (pan; catalog 2920), PKA phospho-substrate (catalog 9621), phospho-ACC (S79; catalog 3661), total ACC (catalog 3676), phospho-AMPK (T172; catalog 2535), total AMPK (catalog 5831), and ADIPOQ total monomer (catalog 2789) were from Cell Signaling Technology. ADIPOQ HMW/LMW (catalog 5901) was from Biovision. Tyrosine hydroxylase (catalog T1299) and UCP1 (catalog U6382) were from Sigma-Aldrich. $\beta$-Actin (catalog sc-47778) was from Santa Cruz Biotechnology. PGC1- $\alpha$ (catalog AB3242) was from EMD Millipore. IP6K1 (catalog GTX103949) was from Genetex.

Enzymes and proteins. LKB1 only (catalog L15-30G), unphosphorylated/inactive AMPK $(\alpha 2 / \beta 1 / \gamma 1)$ (catalog P48-14H), active CaMKK $\beta$ (catalog C18-10G), and phosphorylated/active AMPK (catalog P48-10H) were from SignalChem. Unphosphorylated/inactive AMPK $\alpha 2$ only (catalog H00005563-P01) was from Abnova. LKB1-MO25/STRAD $\alpha$ (cata$\log 14-596)$ was from EMD Millipore. Insulin was from Novo Nordisk.

Kits. The Vectastain Elite ABC kit was from Vector Laboratories. The BCA protein assay kit was from Pierce Biotechnology. The insulin ELISA was from Crystal Chem Inc.

siRNA. AMPK $\alpha$ (catalog sc-45313) and control (catalog sc-37007) siRNAs were from Santa Cruz Biotechnology.

Protease plus phosphatase inhibitor tablets and glutathione agarose. Protease plus phosphatase inhibitor tablets and glutathione agarose were from Thermo Scientific. Unless otherwise stated, all other chemicals were purchased from Sigma-Aldrich.

Animals. Mice were housed in 12-hour light/12-hour dark cycles at $23^{\circ} \mathrm{C}$ and were fed standard chow diet (CD), unless otherwise stated. Two-month-old male mice fed a CD (2018SX; Harlan Laboratories) or a HFD (60\% kcal HFD for indicated periods; S3282; Bio-Serv) were used in the studies. For tissue collection, mice were euthanized by carbon dioxide asphyxiation (ARC Facility).

Generation of AdKO mice. The Ip6k1 fl/ll mouse was previously described (68) and was provided by Solomon Snyder (Johns Hopkins University, Baltimore, Maryland, USA). Details of the strategy to target the Ip $6 k 1$ locus are mentioned in a previous literature (68). Briefly, the mouse Ip $6 k 1$ gene has 6 exons, of which exon 1 is exclusively noncoding (68). The start codon is located in exon 2, and the stop codon is located in exon 6. Exon 6 encodes the C-terminal region of Ip $6 k 1$ (amino acids 265-433), and the 3 '-UTR of the mRNA. Ip6k1 targeting was achieved by deletion of the coding region of exon 6 , including the exon 6 splice site. The resulting transcript lacks exon 6 (including the 3 '-UTR) and therefore is unstable, resulting in a complete knockout.

To generate adipocyte-specific Ip $6 k 1 \mathrm{KO}$ mice, the following mice were purchased from The Jackson Laboratory: FLP (neomycin) deleter (stock 009086); Adipoq-Cre (stock 010803). FRT sites flanking the neomycin resistance gene facilitate its removal by FLP recombinase, and loxP sites facilitate removal of the targeted exon 6 by Cre recombinase. $I p 6 k 1^{f / f l}$ mice were mated with mice expressing FLP recombinase (JAX stock 009086) to excise the neomycin resistance gene to generate $I p 6 k 1^{\text {flpped/fpped }}$ mice. Genotyping was performed by PCR using primer sequences from exon 6 flanking the neomycin resistance gene and FRT sites. Homozygous Ip $6 k 1^{1 \text { tpped } / f p p e d}$ mice were crossed with the transgenic mice expressing Cre recombinase under the control of the ADIPOQ promoter (JAX stock 010803). Offspring inheriting both the 
targeted allele and the Cre transgene (Adipoq-Cre-Ip6k1 flppedffpped) were crossed with $I p 6 k 1^{\text {flppedfflpped }}$ mice to yield Ip6k1 $1^{\text {fpped/flpped }}$ Cre adipocytespecific knockout mice (AdKOs). AdKOs were verified by PCR with Cre primer sequences and primers flanking LoxP sites and exon 6 using DNA extracted from their tails. Male mice were used along with their LoxP (Ip6k1 $\left.1^{\text {fpped/flpped }}\right)$ littermate controls unless otherwise indicated.

Exposure to cold temperature. Chronic exposure was performed following standard procedure (87). Briefly, 2-month-old LoxPs and AdKOs were placed in a cold room $\left(5^{\circ} \mathrm{C}\right)$. After 6 days of cold exposure, mice were sacrificed and tissues were collected for histology and protein analysis.

To induce expenditure of stored energy under cold conditions to maintain body temperature, mice were simultaneously fasted and exposed to cold for indicated time periods (88). Rectal temperature was measured at indicated time points using a mouse-specific rectal probe (RET-3) and a TH-5 thermometer (Physitemp).

Exposure to thermoneutral temperature. Animals were exposed to thermoneutral temperature following standard procedure (89). Briefly, weaned mice ( 22 days old) were introduced to a $30^{\circ} \mathrm{C}$ room. After 1 week of acclimatization, mice were fed a HFD for the indicated period of time.

Food intake studies. Food intake was monitored in HFD-fed LoxPs and AdKOs (at the onset of HFD) using BioDAQ instrumentation (Research diet) following the vendor's recommendations. Mice were acclimatized for 3 days. Afterward, food consumption of each mouse was recorded for 5 consecutive days. A meal is defined as food intake of $0.1 \mathrm{~g}$ or more within 900 seconds, which is the gap between feeding bouts. Data represent the average daily intake. Average values are compared by 2-tailed Student's $t$ test to determine significance.

Comprehensive Laboratory Monitoring System. Mice were placed individually in metabolic cages with a precise thermostatic control in a Comprehensive Laboratory Monitoring System (CLAMS; Columbus Instruments) and were acclimatized at ambient temperature for 2 days. Afterwards, $\mathrm{VO}_{2}, \mathrm{VCO}_{2}$, and spontaneous locomotor activity were measured for indicated time periods. Cold-induced oxygen consumption was assessed by changing of the temperature from ambient to $5^{\circ} \mathrm{C}$ for 24 hours followed by 6 hours of fasting. Respiratory exchange ratio (RER) and energy expenditure (EE) were calculated using the following equations: $\mathrm{RER}=\mathrm{VCO}_{2} / \mathrm{VO}_{2} ; \mathrm{EE}(\mathrm{kcal} / \mathrm{h})=(3.815$ $+1.232 \times \mathrm{RER}) \times \mathrm{VO}_{2}$. Values were normalized by lean body mass.

Calculation of $\mathrm{VO}_{2}$ used for carbohydrate or fat oxidation. Based on mean RER values, the exact percentage of $\mathrm{VO}_{2}$ consumed for carbohydrate and fat oxidation can be determined (72). For example, RER 1.00 means $100 \%$ carbohydrate, whereas RER 0.7 indicates $100 \%$ fat consumption. A complete chart is presented in the literature (72). Accordingly, we calculated $\mathrm{VO}_{2}$ consumed by carbohydrate and fat for AdKOs and LoxPs under various conditions. Significance was calculated by 2-tailed Student's $t$ test.

$N M R$. Fat, lean, and fluid mass of CD- and HFD-fed mice was measured using the Minispec LF-NMR (Brucker Optics) analyzer.

Blood glucose measurement. Blood glucose in ad libitum and overnight-fasted HFD-fed mice was measured using a glucometer (Bayer) with glucose testing strips as described previously (58). Significance is calculated by 2-way ANOVA.

Glucose tolerance test. GTT was performed after 14 weeks in HFDfed AdKOs under various temperature conditions unless otherwise stated. Glucose ( $2 \mathrm{~g} / \mathrm{kg}$ body weight; i.p.) was injected into overnightfasted mice. After injection, blood was taken by puncturing of the tail vein, and glucose levels were measured using a glucometer at indicated time periods. Blood glucose was also measured before the injection (time point 0 ).

Insulin tolerance test. After 14 weeks of $\mathrm{HFD}$ at $23^{\circ} \mathrm{C}$, ITT was performed in AdKOs and LoxPs. After 6 hours of fasting in the morning, human recombinant insulin ( $0.75 \mathrm{U} / \mathrm{kg}$ body weight; i.p.) was injected. Blood glucose levels were measured as described above.

TNP injection in mice. WT C57BL/6 mice were fed a HFD for 4 weeks. Thereafter, vehicle or TNP $(10 \mathrm{mg} / \mathrm{kg}$ body weight; daily; i.p. $)$ ( $n=5$ per group) was injected for 7 weeks. Laboratory-synthesized TNP was dissolved in DMSO/Tween-80/water (1:1:8). Weekly body weight was measured. After 4 weeks of injection, oxygen consumption was measured as described above. GTT and ITT were performed on the fifth and sixth weeks of TNP treatment, respectively. In an independent experiment ( $n=3$ per group), mice were fed a HFD and treated with TNP as described above. After 7 weeks, they were cold-exposed for 4 days. To monitor AMPK activation, a single dose ( $20 \mathrm{mg} / \mathrm{kg}$ body weight) of TNP was injected in DIO mice. After the treatment, mice were fasted for 5 hours, after which they were sacrificed for tissue collection.

Plasma insulin measurement. Blood was collected from LoxPs and AdKOs after 14 weeks of HFD feeding in fed and 6-hour-fasted conditions into a $\mathrm{K}_{2}$ EDTA-containing tube, and plasma was isolated by centrifuge at $4^{\circ} \mathrm{C}$ for 15 minutes at 2,000 $\mathrm{g}$. Insulin concentrations were determined using an ultrasensitive mouse ELISA kit.

Histological and immunohistochemical analyses of adipose tissue. Tissues isolated from various adipose depots were fixed overnight in $10 \%$ neutral-buffered formalin. Samples were processed in a dehydrating ethanol gradient, followed by xylene incubation and paraffin embedding. Serial $8-\mu \mathrm{m}$ sections were used for H\&E staining, or for UCP1 immunohistochemical analysis. For immunohistochemistry, primary antibody was detected by Vectastain Elite ABC kit and diaminobenzidine reagent using the manufacturer's instructions.

Analyses of plasma ADIPOQ. ADIPOQ complexes in the plasma were monitored following standard protocol (90). Briefly, equal volume of plasma was mixed with Laemmli buffer without $\beta$-mercaptoethanol, and proteins were resolved in $4 \%-15 \%$ SDS-PAGE. Primary antibody against ADIPOQ complex (Biovision) was used to detect the high-molecular weight (HMW) and low-molecular weight (LMW) complexes. To quantify total ADIPOQ, Laemmli buffer containing $\beta$-mercaptoethanol was used, and samples were heated at $95^{\circ} \mathrm{C}$ for 10 minutes. Primary antibody against monomeric ADIPOQ (Cell Signaling Technology) was used. The nitrocellulose membranes were ponceau-stained to confirm sample loading.

RNA isolation and quantitative RT-PCR. RNA isolation was done using the traditional Trizol method. Quantitative gene expression was performed by 2-step quantitative RT-PCR (qRT-PCR) using Applied Biosystems kit and TaqMan probes. mRNA expression levels were determined using the $\Delta \Delta \mathrm{CT}$ method and normalized to the housekeeping gene $\beta$-actin.

SVF culture and beige adipocyte differentiation. Subcutaneous inguinal fat from 6- to 8-week-old mice ( $n=6$ per group) was used for isolating stromal vascular cells as previously described (91). Cells were cultured in DMEM/F12 containing 10\% FBS and penicillin/streptomycin. Ninetyfive to ninety-seven percent confluent cells were differentiated with a medium containing $5 \mu \mathrm{g} / \mathrm{ml}$ insulin, $125 \mu \mathrm{M}$ indomethacin, $2 \mu \mathrm{g} / \mathrm{ml}$ dexamethasone, $0.5 \mathrm{mM}$ 3-isobutyl-1-methylxanthine, $0.5 \mu \mathrm{M}$ rosiglitazone, and $1 \mathrm{nM} \mathrm{3,3',5-triiodo-L-thyronine} \mathrm{(T3).} \mathrm{After} 2$ days, medium was replaced with maintenance medium containing $5 \mu \mathrm{g} / \mathrm{ml}$ insulin and 
$1 \mathrm{nM}$ T3. Medium was refreshed every 2-3 days until the cells were fully differentiated at day 7. For AICAR and TNP treatments, differentiated beige adipocytes were incubated overnight with $1 \mathrm{mM}$ AICAR or $5 \mu \mathrm{M}$ TNP. Cells were washed with PBS and harvested for qRT-PCR analyses.

Mitochondrial respiration analysis. For measuring mitochondrial respiration, SVF cells were seeded into $96 \mathrm{XF}$ plates and differentiated to beige adipocytes. Oxygen consumption rate (OCR) and extracellular acidification were measured by Seahorse Extracellular Flux Analyzer (Seahorse Bioscience) in XF assay medium supplemented with $5 \mathrm{mM}$ pyruvate and $2.5 \mathrm{mM}$ glucose. After baseline measurements, 3 sequential injections were applied to the cells as indicated: oligomy$\operatorname{cin}(1 \mu \mathrm{g} / \mathrm{ml})$, FCCP $(1 \mu \mathrm{M})$, and antimycin A $(0.8 \mu \mathrm{M})$ combined with rotenone $(3 \mu \mathrm{M})$. Basal, uncoupled, and maximal OCRs were calculated by averaging of values from each phase.

Signaling studies. SVF isolated from IWAT was differentiated to beige adipocytes as described above, and the cells were lysed by addition of a lysis buffer (20 mM Tris pH 7.4; $150 \mathrm{mM} \mathrm{NaCl}$; 1\% Triton $\mathrm{X}-100)$ containing a protease-phosphatase inhibitor tablet. Total protein was quantified using a BCA protein assay kit (Pierce Biotechnology). Equal amounts of total protein were loaded on SDS-PAGE. Various proteins and their phosphorylation levels were monitored by immunoblotting following standard procedures (58). Beige adipocytes or 3T3L1 preadipocytes were treated with indicated concentrations of TNP under basal and/or 2-hour-glucose-starved conditions. HEK293 cells were transfected using Polyfect transfection reagent (Qiagen) (58). Forty-eight hours after transfection, cells were glucose-starved for 2 hours followed by glucose reintroduction $(25 \mathrm{mM})$ for $30 \mathrm{~min}$ utes. After the experiments, cells were lysed and samples were processed as above for immunoblot analysis.

Coimmunoprecipitation studies. Three milligrams of lysates from IWAT was used to immunoprecipitate endogenous IP6K1 following standard protocol (Cell Signaling Technology). The samples were resolved by SDS-PAGE, and coimmunoprecipitation of AMPK $\alpha$ was determined using anti-AMPK $\alpha$ antibody. Loading of AMPK $\alpha$ and IP6K1 was detected in $1 \%$ of the input samples used in immunoprecipitation studies.

GST pulldown assay. To determine the AMPK $\alpha$ binding motif in IP6K1, HEK 293 cells at about $80 \%$ confluence were transfected with a series of GST-tagged Ip6k1 deletion mutants using Polyfect transfection reagent. Forty-eight hours after transfection, cells were washed with PBS and lysed. Glutathione agarose was used to pull down GSTtagged IP6K1 and its mutants. Samples were washed 3 times with wash buffer. Afterward, beads were boiled with lithium dodecyl sodium (LDS) loading buffer and resolved in SDS-PAGE. IP6K1 and AMPK $\alpha$ were detected by GST- and AMPK $\alpha$-specific antibodies, respectively. To test effects of TNP on the interaction of endogenous AMPK $\alpha$ and GST-IP6K1, cells transfected with indicated GST-constructs for 48 hours were treated with $5 \mu \mathrm{M}$ TNP for 2 hours. One milligram of the lysate was used for GST pulldown assay.

AMPKa knockdown by RNA interference. Expression of endogenous AMPK $\alpha 1$ and $\alpha 2$ isoforms was diminished by introducing a pool of 3 siRNAs targeting AMPK $\alpha$ (92). Briefly, 2 and 4 days after differentiation, cells were transfected with $100 \mathrm{nM}$ scrambled or AMPK $\alpha$ specific siRNAs using jetPRIME reagent following the manufacturer's instructions. Cells were washed with PBS and harvested on day 6 for qRT-PCR or immunoblotting analysis.

Effects of inositol phosphates on AMPK in vitro. The effects of various inositol phosphates and IP6K1 on LKB1-mediated AMPK activ- ity were determined using either LKB1-only or LKB1-complex (with MO25 and STRAD) on the unphosphorylated/inactive AMPK ( $\alpha 2 /$ $\beta 1 / \gamma 1)$ substrate. Briefly, IP3 [Ins(1,4,5)P3], IP4 [Ins(1,3,4,5)P4], IP5 [Ins(1,3,4,5,6)P5], IP5* [Ins(2,3,4,5,6)P5] (43), IP6 [Ins(1,2,3,4,5,6)P6], 5-IP7, and purified catalytically active and inactive IP6K1 (49) were added at indicated concentrations in a reaction buffer $(25 \mathrm{mM}$ Tris $\mathrm{pH}$ 7.2, $5 \mathrm{mM} \mathrm{MgCl}_{2}$, and $2.5 \mathrm{mM} \mathrm{DTT}$ ) containing AMPK $\alpha 2 / \beta 1 / \gamma 1$ (100 $\mathrm{ng} /$ reaction), LKB1-only (120 ng/reaction), and ATP $(100 \mu \mathrm{M})$ to initiate the reaction at $30^{\circ} \mathrm{C}$. The total reaction volume was $25 \mu \mathrm{l}$. After 30 minutes, the reaction was stopped by addition of EDTA-containing LDS gel loading buffer and boiled at $95^{\circ} \mathrm{C}$. SDS-PAGE was run and the extent of AMPK phosphorylation (T172) was detected by immunoblotting. All the inositol phosphates other than IP5 ${ }^{*}$ were purchased from Cayman Chemical. IP5* was from Enzo Life Science.

To determine IP6 and IP6K1's effects on AMPK phosphorylation mediated by LKB1-complex (with MO25 and STRAD $\alpha$ ), LKB1 concentration for the assay was determined to be $10 \mathrm{ng} / \mathrm{reaction}$, as opposed to $120 \mathrm{ng} /$ reaction of LKB1-only. Everything else was kept similar to the above assay.

To compare IP6's effects on LKB1-mediated AMPK $(\alpha 2 / \beta 1 / \gamma 1)$ and AMPK2, equimolar concentrations of AMPK ( $25 \mathrm{nM})$ were used as substrates. In the assay of CaMKK2-mediated AMPK activity, active CaMKK $\beta$ (10 ng/reaction) was used instead of LKB1, and the rest was similar as above. Except for 5-IP7, which was chemically synthesized following standard procedure (93), all the other inositol phosphates were purchased.

AMPK activity assay in vitro. AMPK activity was determined using the SAMS peptide (SAMStide; Anaspec) as a substrate. The assay buffer consisted of $25 \mathrm{mM}$ Tris ( $\mathrm{pH} 7.2$ ), $5 \mathrm{mM} \mathrm{MgCl}, 2.5 \mathrm{mM}$ DTT, $200 \mathrm{ng} /$ $\mu$ l SAMStide, and active AMPK $(\alpha 2 / \beta 1 / \gamma 1 ; 1.5 \mathrm{nM})$ in the presence of indicated concentrations of AMP and IP6 in a volume of $20 \mu \mathrm{l}$. To initiate the reaction, $5 \mu \mathrm{l}$ of the ATP cocktail (cocktail: $150 \mu \mathrm{l} 10 \mathrm{mM}$ ATP, $100 \mu \mathrm{l} 10 \mu \mathrm{Ci} / \mu \mathrm{l}{ }^{33} \mathrm{P}-\gamma \mathrm{ATP}$, and $5.75 \mathrm{ml}$ assay buffer) was added and then incubated at $30^{\circ} \mathrm{C}$ for 30 minutes. The reaction was terminated by spotting of $20-\mu \mathrm{l}$ reaction mixtures onto individual precut strips of P81 phosphocellulose paper. The papers were washed in $1 \%$ phosphoric acid with constant gentle stirring. The radioactivity on the P81 paper was counted. The kinase-specific activity was presented as picomoles per minute per microgram.

Statistics. Trial/prior experiments were used to determine sample size with ample statistical power. Animals were excluded from experiments if they showed any signs of sickness. Numbers of mice $(n)$ used in experiments are indicated in the legends. Immunoblots were quantified using Image J software (NIH). For multiple comparisons, 2-way ANOVA with Holm-Šidák multiple comparison test was used. For 2 independent data sets, 2-tailed Student's $t$ test was used. Data are presented as mean \pm SEM $\left({ }^{\S} P<0.0001,{ }^{*} P<0.001,{ }^{* *} P<0.01\right.$, and $\left.{ }^{*} P<0.05\right)$. Statistical significance was calculated in GraphPad Prism, version 6 . The data show normal variance.

See complete unedited blots in the supplemental material.

Study approval. Animal care and experimentation were approved by the Scripps Florida Institutional Animal Care and Use Committee.

\section{Author contributions}

AC conceived and supervised the project; QZ generated AdKO mice; $\mathrm{QZ}$ and AR maintained mouse colonies; QZ performed the majority of the experiments; S. Ghoshal performed pharmacologic experi- 
ments; AR and S. Gao provided technical support; AA and TMK synthesized TNP; JCB supplied chemically synthesized 5-IP7; AC and $\mathrm{QZ}$ analyzed and interpreted data; AC wrote the manuscript.

\section{Acknowledgments}

We sincerely thank the Snyder laboratory at Johns Hopkins University School of Medicine for sharing $I p 6 k 1^{f / f l}$ mice and other reagents. We also thank Melissa Kazantzis, Montina Van Meter, and Xiangyang Xie for helping in CLAMS, food intake, and histological and qRT-PCR analyses; Agustin Jimenez-Colon and Dana Mulvaney for assistance in animal maintenance; Andras Kern and Roy Smith for sharing qRT-PCR probes; Andrew Butler for his help in analyzing the energy expenditure data; and the Scripps Research Institute Depart- ment of Metabolism and Aging for sharing reagents and instruments. The work was supported by NIH grant R01DK103746 and a Scripps Research Institute startup fund 1-31564 to Anutosh Chakraborty.

Address correspondence to: Anutosh Chakraborty, Department of Metabolism and Aging, The Scripps Research Institute, 130 Scripps Way, Jupiter, Florida 33458, USA. Phone: 561.228.2426; E-mail: achakrab@scripps.edu.

AR's present address is: Ophtec USA Inc., Boca Raton, Florida, USA. S. Gao's present address is: Department of Pediatrics, University of Alberta, Edmonton, Canada. AA's present address is: Department of Chemistry, University of Cambridge, Cambridge, United Kingdom.
1. Tseng YH, Cypess AM, Kahn CR. Cellular bioenergetics as a target for obesity therapy. Nat Rev Drug Discov. 2010;9(6):465-482.

2. Kozak LP, Koza RA, Anunciado-Koza R. Brown fat thermogenesis and body weight regulation in mice: relevance to humans. Int JObes (Lond). 2010;34(suppl 1):S23-S27.

3. Cinti S. Transdifferentiation properties of adipocytes in the adipose organ. Am J Physiol Endocrinol Metab. 2009;297(5):E977-E986.

4. Rosen ED, Spiegelman BM. What we talk about when we talk about fat. Cell. 2014;156(1-2):20-44.

5. Whittle A, Relat-Pardo J, Vidal-Puig A. Pharmacological strategies for targeting BAT thermogenesis. Trends Pharmacol Sci. 2013;34(6):347-355.

6. Chechi K, Nedergaard J, Richard D. Brown adipose tissue as an anti-obesity tissue in humans. Obes Rev. 2014;15(2):92-106.

7. Cypess AM, Kahn CR. Brown fat as a therapy for obesity and diabetes. Curr Opin Endocrinol Diabetes Obes. 2010;17(2):143-149.

8. Sidossis L, Kajimura S. Brown and beige fat in humans: thermogenic adipocytes that control energy and glucose homeostasis. J Clin Invest. 2015;125(2):478-486.

9. Hany TF, Gharehpapagh E, Kamel EM, Buck A, Himms-Hagen J, von Schulthess GK. Brown adipose tissue: a factor to consider in symmetrical tracer uptake in the neck and upper chest region. Eur J Nucl Med Mol Imaging. 2002;29(10):1393-1398.

10. van Marken Lichtenbelt WD, et al. Cold-activated brown adipose tissue in healthy men. $\mathrm{NEnglJ}$ Med. 2009;360(15):1500-1508.

11. Cypess AM, et al. Identification and importance of brown adipose tissue in adult humans. $N$ EnglJ Med. 2009;360(15):1509-1517.

12. Saito M, et al. High incidence of metabolically active brown adipose tissue in healthy adult humans: effects of cold exposure and adiposity. Diabetes. 2009;58(7):1526-1531.

13. Chondronikola $\mathrm{M}$, et al. Brown adipose tissue improves whole-body glucose homeostasis and insulin sensitivity in humans. Diabetes. 2014;63(12):4089-4099.

14. Orava J, et al. Blunted metabolic responses to cold and insulin stimulation in brown adipose tissue of obese humans. Obesity (Silver Spring). 2013;21(11):2279-2287.

15. Harms M, Seale P. Brown and beige fat: development, function and therapeutic potential. Nat
Med. 2013;19(10):1252-1263.

16. Collins S. A heart-adipose tissue connection in the regulation of energy metabolism. Nat Rev Endocrinol. 2014;10(3):157-163.

17. Scarpulla RC, Vega RB, Kelly DP. Transcriptional integration of mitochondrial biogenesis. Trends Endocrinol Metab. 2012;23(9):459-466.

18. Finck BN, Kelly DP. PGC-1 coactivators: inducible regulators of energy metabolism in health and disease. J Clin Invest. 2006;116(3):615-622.

19. Mulligan JD, Gonzalez AA, Stewart AM, Carey HV, Saupe KW. Upregulation of AMPK during cold exposure occurs via distinct mechanisms in brown and white adipose tissue of the mouse. J Physiol (Lond). 2007;580(pt 2):677-684.

20. Oliveira RL, et al. Cold-induced PGC-1alpha expression modulates muscle glucose uptake through an insulin receptor/Akt-independent, AMPK-dependent pathway. Am J Physiol Endocrinol Metab. 2004;287(4):E686-E695.

21. Steinberg GR, Kemp BE. AMPK in health and disease. Physiol Rev. 2009;89(3):1025-1078.

22. Inokuma K, Ogura-Okamatsu Y, Toda C, Kimura $\mathrm{K}$, Yamashita H, Saito M. Uncoupling protein 1 is necessary for norepinephrine-induced glucose utilization in brown adipose tissue. Diabetes. 2005;54(5):1385-1391.

23. Klaus S, Keipert S, Rossmeisl M, Kopecky J. Augmenting energy expenditure by mitochondrial uncoupling: a role of AMP-activated protein kinase. Genes Nutr. 2012;7(3):369-386.

24. Yan M, et al. Chronic AMPK activation via loss of FLCN induces functional beige adipose tissue through PGC-1 $\alpha /$ ERR $\alpha$. Genes Dev. 2016;30(9):1034-1046.

25. Pulinilkunnil T, et al. Adrenergic regulation of AMP-activated protein kinase in brown adipose tissue in vivo. J Biol Chem. 2011;286(11):8798-8809.

26. Zhang Z, et al. Berberine activates thermogenesis in white and brown adipose tissue. Nat Commun. 2014;5:5493.

27. Zhang H, et al. MicroRNA-455 regulates brown adipogenesis via a novel HIF1an-AMPK-PGC1 $\alpha$ signaling network. EMBO Rep. 2015;16(10):1378-1393.

28. Doménech E, et al. AMPK and PFKFB3 mediate glycolysis and survival in response to mitophagy during mitotic arrest. Nat Cell Biol. 2015;17(10):1304-1316.

29. Cantó C, Auwerx J. PGC-1 $\alpha$, SIRT1 and AMPK, an energy sensing network that controls energy expen- diture. Curr Opin Lipidol. 2009;20(2):98-105.

30. Hardie DG, Ross FA, Hawley SA. AMPK: a nutrient and energy sensor that maintains energy homeostasis. Nat Rev Mol Cell Biol. 2012;13(4):251-262.

31. O’Neill HM, Holloway GP, Steinberg GR. AMPK regulation of fatty acid metabolism and mitochondrial biogenesis: implications for obesity. Mol Cell Endocrinol. 2013;366(2):135-151.

32. Gaidhu MP, Frontini A, Hung S, Pistor K, Cinti S, Ceddia RB. Chronic AMP-kinase activation with AICAR reduces adiposity by remodeling adipocyte metabolism and increasing leptin sensitivity. JLipid Res. 2011;52(9):1702-1711.

33. Smith AC, Bruce CR, Dyck DJ. AMP kinase activation with AICAR simultaneously increases fatty acid and glucose oxidation in resting rat soleus muscle. J Physiol (Lond). 2005;565(pt 2):537-546.

34. Wakil SJ, Abu-Elheiga LA. Fatty acid metabolism: target for metabolic syndrome. J Lipid Res. 2009;50(suppl):S138-S143.

35. Mihaylova MM, Shaw RJ. The AMPK signalling pathway coordinates cell growth, autophagy and metabolism. Nat Cell Biol. 2011;13(9):1016-1023.

36. Alessi DR, Sakamoto K, Bayascas JR. LKB1dependent signaling pathways. Annu Rev Biochem. 2006;75:137-163.

37. Hardie DG. AMPK: positive and negative regulation, and its role in whole-body energy homeostasis. Curr Opin Cell Biol. 2015;33:1-7.

38. Berridge MJ, Lipp P, Bootman MD. The versatility and universality of calcium signalling. Nat Rev Mol Cell Biol. 2000;1(1):11-21.

39. Stephens L, et al. The detection, purification, structural characterization, and metabolism of diphosphoinositol pentakisphosphate(s) and bisdiphosphoinositol tetrakisphosphate(s). J Biol Chem. 1993;268(6):4009-4015.

40. Menniti FS, Miller RN, Putney JW, Shears SB. Turnover of inositol polyphosphate pyrophosphates in pancreatoma cells. J Biol Chem. 1993;268(6):3850-3856.

41. Shears SB. Diphosphoinositol polyphosphates: metabolic messengers? Mol Pharmacol. 2009;76(2):236-252.

42. Wilson MS, Livermore TM, Saiardi A. Inositol pyrophosphates: between signalling and metabolism. Biochem J. 2013;452(3):369-379.

43. Wundenberg T, Grabinski N, Lin H, Mayr GW. Discovery of InsP6-kinases as InsP6-dephosphor- 
ylating enzymes provides a new mechanism of cytosolic InsP6 degradation driven by the cellular ATP/ADP ratio. Biochem J. 2014;462(1):173-184.

44. York JD. Regulation of nuclear processes by inositol polyphosphates. Biochim Biophys Acta. 2006;1761(5-6):552-559.

45. Chakraborty A, Kim S, Snyder SH. Inositol pyrophosphates as mammalian cell signals. Sci Signal. 2011;4(188):re1.

46. Rao F, et al. Inositol pyrophosphates mediate the DNA-PK/ATM-p53 cell death pathway by regulating CK2 phosphorylation of Tti1/Tel2. Mol Cell. 2014;54(1):119-132.

47. Thota SG, Bhandari R. The emerging roles of inositol pyrophosphates in eukaryotic cell physiology. J Biosci. 2015;40(3):593-605.

48. Lee TS, et al. Inositol pyrophosphates inhibit synaptotagmin-dependent exocytosis. Proc Natl Acad Sci U S A. 2016;113(29):8314-8319.

49. Chakraborty A, Latapy C, Xu J, Snyder SH, Beaulieu JM. Inositol hexakisphosphate kinase-1 regulates behavioral responses via GSK3 signaling pathways. Mol Psychiatry. 2014;19(3):284-293.

50. Luo HR, et al. GRAB: a physiologic guanine nucleotide exchange factor for Rab3A, which interacts with inositol hexakisphosphate kinase. Neuron. 2001;31(3):439-451.

51. Fu C, et al. Inositol Hexakisphosphate Kinase-3 regulates the morphology and synapse formation of cerebellar purkinje cells via spectrin/adducin. J Neurosci. 2015;35(31):11056-11067.

52. Ghoshal S, Tyagi R, Zhu Q, Chakraborty A. Inositol hexakisphosphate kinase-1 interacts with perilipin1 to modulate lipolysis. Int J Biochem Cell Biol. 2016;78:149-155.

53. Barker CJ, Illies C, Gaboardi GC, Berggren PO. Inositol pyrophosphates: structure, enzymology and function. Cell Mol Life Sci. 2009;66(24):3851-3871.

54. Morrison BH, Haney R, Lamarre E, Drazba J, Prestwich GD, Lindner DJ. Gene deletion of inositol hexakisphosphate kinase 2 predisposes to aerodigestive tract carcinoma. Oncogene. 2009;28(25):2383-2392.

55. Szijgyarto Z, Garedew A, Azevedo C, Saiardi A. Influence of inositol pyrophosphates on cellular energy dynamics. Science. 2011;334(6057):802-805.

56. Jadav RS, et al. Deletion of inositol hexakisphosphate kinase 1 (IP6K1) reduces cell migration and invasion, conferring protection from aerodigestive tract carcinoma in mice. Cell Signal. 2016;28(8):1124-1136.

57. Illies C, et al. Requirement of inositol pyrophosphates for full exocytotic capacity in pancreatic beta cells. Science. 2007;318(5854):1299-1302.

58. Chakraborty A, et al. Inositol pyrophosphates inhibit Akt signaling, thereby regulating insulin sensitivity and weight gain. Cell. 2010;143(6):897-910.

59. Prasad A, et al. Inositol hexakisphosphate kinase 1 regulates neutrophil function in innate immunity by inhibiting phosphatidylinositol$(3,4,5)$-trisphosphate signaling. Nat Immunol. 2011;12(8):752-760

60. Wu M, Dul BE, Trevisan AJ, Fiedler D. Synthesis and characterization of non-hydrolysable diphosphoinositol polyphosphate second messengers. Chem Sci. 2013;4(1):405-410.

61. Zhang Z, et al. Inositol pyrophosphates mediate the effects of aging on bone marrow mesenchymal stem cells by inhibiting Akt signaling. Stem Cell Res Ther. 2014;5(2):33.

62. Burton A, Azevedo C, Andreassi C, Riccio A, Saiardi A. Inositol pyrophosphates regulate JMJD2C-dependent histone demethylation. Proc Natl Acad Sci U S A. 2013;110(47):18970-18975.

63. Chang YT, et al. Purine-based inhibitors of inositol-1,4,5-trisphosphate-3-kinase. Chembiochem. 2002;3(9):897-901.

64. Padmanabhan U, Dollins DE, Fridy PC, York JD, Downes CP. Characterization of a selective inhibitor of inositol hexakisphosphate kinases: use in defining biological roles and metabolic relationships of inositol pyrophosphates. J Biol Chem. 2009;284(16):10571-10582.

65. Wang H, Falck JR, Hall TM, Shears SB. Structura basis for an inositol pyrophosphate kinase surmounting phosphate crowding. Nat Chem Biol. 2012;8(1):111-116

66. Sun D, et al. Oncostatin M (OSM) protects against cardiac ischaemia/reperfusion injury in diabetic mice by regulating apoptosis, mitochondrial biogenesis and insulin sensitivity. JCell Mol Med. 2015;19(6):1296-1307.

67. Sarmah B, Wente SR. Inositol hexakisphosphate kinase-2 acts as an effector of the vertebrate Hedgehog pathway. Proc Natl Acad Sci U S A. 2010;107(46):19921-19926.

68. Bhandari R, Juluri KR, Resnick AC, Snyder $\mathrm{SH}$. Gene deletion of inositol hexakisphosphate kinase 1 reveals inositol pyrophosphate regulation of insulin secretion, growth, and spermiogenesis. Proc Natl Acad Sci U S A. 2008;105(7):2349-2353.

69. Boucher J, Kleinridders A, Kahn CR. Insulin receptor signaling in normal and insulinresistant states. Cold Spring Harb Perspect Biol. 2014;6(1):a009191.

70. Mackenzie RW, Elliott BT. Akt/PKB activation and insulin signaling: a novel insulin signaling pathway in the treatment of type 2 diabetes. Diabetes Metab Syndr Obes. 2014;7:55-64.

71. Ghosh S, et al. Inositol hexakisphosphate kinase 1 maintains hemostasis in mice by regulating platelet polyphosphate levels. Blood. 2013;122(8):1478-1486.

72. Lusk G. Animal calorimetry: analysis of the oxidation of mixtures of carbohydrate and fat. J Biol Chem. 1924;59(12):41-42.

73. Shetty S, Kusminski CM, Scherer PE. Adiponectin in health and disease: evaluation of adiponectintargeted drug development strategies. Trends Pharmacol Sci. 2009;30(5):234-239.

74. Hinoi E, et al. PI3K/Akt is involved in brown adipogenesis mediated by growth differentiation factor-5 in association with activation of the Smad pathway. Biochem Biophys Res Commun. 2014;450(1):255-260.

75. Valverde AM, et al. Insulin-induced up-regulated uncoupling protein-1 expression is mediated by insulin receptor substrate 1 through the phosphatidylinositol 3-kinase/Akt signaling pathway in fetal brown adipocytes. J Biol Chem 2003;278(12):10221-10231.

76. Hui X, et al. Adiponectin enhances cold-induced browning of subcutaneous adipose tissue via promoting M2 macrophage proliferation. Cell Metab.
2015;22(2):279-290.

77. Ghoshal S, et al. TNP [N2-(m-Trifluorobenzyl), N6-(p-nitrobenzyl)purine] ameliorates diet induced obesity and insulin resistance via inhibition of the IP6K1 pathway [published online ahead of print August 21, 2016]. Mol Metab. doi:10.1016/j.molmet.2016.08.008.

78. Farese RV, Sajan MP, Standaert ML. Insulinsensitive protein kinases (atypical protein kinase $\mathrm{C}$ and protein kinase $\mathrm{B} / \mathrm{Akt}$ ): actions and defects in obesity and type II diabetes. Exp Biol Med (Maywood). 2005;230(9):593-605.

79. Wang Q, et al. Autoinhibition of Bruton's tyrosine kinase (Btk) and activation by soluble inositol hexakisphosphate. Elife. 2015;4:e06074.

80. Rao F, et al. Inositol pyrophosphates promote tumor growth and metastasis by antagonizing liver kinase B1. Proc Natl Acad Sci U S A. 2015;112(6):1773-1778.

81. Xie Z, Dong Y, Zhang J, Scholz R, Neumann D, Zou MH. Identification of the serine 307 of LKB1 as a novel phosphorylation site essential for its nucleocytoplasmic transport and endothelial cell angiogenesis. Mol Cell Biol. 2009;29(13):3582-3596.

82. Vingtdeux V, Chandakkar P, Zhao H, Davies P, Marambaud P. Small-molecule activators of AMP-activated protein kinase (AMPK), RSVA314 and RSVA405, inhibit adipogenesis. Mol Med. 2011;17(9-10):1022-1030.

83. Xiao B, et al. Structural basis of AMPK regulation by small molecule activators. Nat Commun. 2013;4:3017.

84. Bang S, Chen Y, Ahima RS, Kim SF. Convergence of IPMK and LKB1-AMPK signaling pathways on metformin action. Mol Endocrinol. 2014;28(7):1186-1193.

85. Maag D, et al. Inositol polyphosphate multikinase is a physiologic PI3-kinase that activates Akt/PKB. Proc Natl Acad Sci U S A. 2011;108(4):1391-1396.

86. Bang S, et al. AMP-activated protein kinase is physiologically regulated by inositol polyphosphate multikinase. Proc Natl Acad Sci US A. 2012;109(2):616-620.

87. Kong $\mathrm{X}$, et al. IRF4 is a key thermogenic transcriptional partner of PGC-1 $\alpha$. Cell. 2014;158(1):69-83.

88. Chatterjee TK, et al. HDAC9 knockout mice are protected from adipose tissue dysfunction and systemic metabolic disease during high-fat feeding. Diabetes. 2014;63(1):176-187.

89. Petruzzelli M, et al. A switch from white to brown fat increases energy expenditure in cancer-associated cachexia. Cell Metab. 2014;20(3):433-447.

90. Mansuy-Aubert V, et al. Imbalance between neutrophil elastase and its inhibitor $\alpha 1$-antitrypsin in obesity alters insulin sensitivity, inflammation, and energy expenditure. Cell Metab. 2013;17(4):534-548.

91. Aune UL, Ruiz L, Kajimura S. Isolation and differentiation of stromal vascular cells to beige/brite cells. J Vis Exp. 2013;73(73):50191.

92. Kim SJ, Nian C, McIntosh CH. Activation of lipoprotein lipase by glucose-dependent insulinotropic polypeptide in adipocytes. A role for a protein kinase B, LKB1, and AMP-activated protein kinase cascade. JBiol Chem. 2007;282(12):8557-8567.

93. Zhang H, Thompson J, Prestwich GD. A scalable synthesis of the IP7 isomer, 5-PP-Ins $(1,2,3,4,6)$ P5. Org Lett. 2009;11(7):1551-1554. 